hep-th/0202067

KCL-MTH-02-03

\title{
Boundary states for WZW models
}

\author{
Matthias R. Gaberdiel* \\ Department of Mathematics, King's College London \\ Strand, London WC2R 2LS, U.K. \\ and \\ Terry Gannon ${ }^{\star}$ \\ Department of Mathematical Sciences, University of Alberta \\ Edmonton, Alberta, Canada, T6G $2 G 1$
}

\begin{abstract}
The boundary states for a certain class of WZW models are determined. The models include all modular invariants that are associated to a symmetry of the unextended Dynkin diagram. Explicit formulae for the boundary state coefficients are given in each case, and a number of properties of the corresponding NIM-reps are derived.
\end{abstract}

February, 2002

\footnotetext{
* mrg@mth.kcl.ac.uk

* tgannon@math.ualberta.ca
} 


\section{Introduction}

Given a conformal field theory defined on closed Riemann surfaces (a closed string theory) we can ask whether the theory can be defined on Riemann surfaces with boundaries. More precisely we can ask which boundary conditions can be imposed at the various boundaries. From a string theory point of view, this is the question of which open strings can be consistently added to a given closed string theory. In general, rather little is known about when a 'complete' set of boundary conditions can be found. It is believed that for closed string theories that are consistently defined on arbitrary genus surfaces such a solution exists, but not even this is clear. Even if the higher genus property is sufficient, it seems unlikely to be necessary.

One of the conditions that every consistent set of boundary states has to satisfy is the so-called Cardy condition [1]: suppose that the conformal field theory is a rational conformal field theory with respect to some symmetry algebra $\mathcal{A}$, which possesses $N$ irreducible highest weight representations. The Cardy condition then implies that every set of $M$ boundary conditions determines $N M \times M$ matrices of non-negative integers, one for each highest weight representation of $\mathcal{A}$. Furthermore, if the set of boundary conditions is complete in a suitable sense [2], these matrices actually form a (Non-negative Integer Matrix) representation, or NIM-rep for short, of the fusion algebra (see [3, 1 , 5] for an introduction to these matters).

The condition of a representation of the fusion algebra to be a NIM-rep is quite restrictive, and one can therefore attempt to classify all such representations (irrespective of whether they arise from boundary states or not). If modular invariance is indeed a sufficient condition for the construction of the boundary states, the classification of NIMreps then gives a restriction on the possible modular invariant theories. This program has been performed for the case of the WZW models corresponding to $\mathrm{su}(2)$ in [3]. However, in general this approach does not seem to be powerful since there are typically many more NIM-reps than modular invariant conformal field theories [5], the best known examples being the tadpoles of $\mathrm{su}(2)$ [3]. (There are also many modular invariant partition functions that do not have a NIM-rep [5]; however, at least some of them do not define consistent conformal field theories, and it is therefore conceivable that modular invariance is indeed sufficient for the construction of a boundary theory.) In the following we shall call a NIMrep 'physical' if it is compatible with a modular invariant (see the end of section 2 below). If the NIM-rep possesses in addition compatible structures corresponding to the defect 
lines and $3 \mathrm{j}$ - and $6 \mathrm{j}$-symbols (see for example [6]), we shall call it 'fully realised'. Fully realised NIM-reps can be obtained from a subfactor [0],8].

It is generally believed that the conformal field theory whose space of states is described by the diagonal modular invariant defines a consistent conformal field theory that is defined on arbitrary Riemann surfaces. * Given this theory, we can obtain other consistent modular invariant conformal field theories by applying a (global) symmetry transformation to the left-moving degrees of freedom, say. For example, for the case of the WZW models corresponding to the simple Lie algebra $\overline{\mathfrak{g}}$, any symmetry of the (unextended) Dynkin diagram can be employed. Symmetries of the unextended Dynkin diagram exist for $\overline{\mathfrak{g}}=\mathrm{su}(n)$ and $E_{6}$, where they correspond to 'charge conjugation'; for $\overline{\mathfrak{g}}=\mathrm{so}(2 n)$, where they correspond to the 'chirality flip'; and for $\overline{\mathfrak{g}}=\mathrm{so}(8)$ where there is in addition 'triality'.

The boundary states of the usual diagonal modular invariant theory are known in detail and explicitly (they are described by Cardy's formula [1]), but the boundary states of the theories that can be obtained using one of the above symmetries are less well understood. In this paper we shall make a proposal for the boundary states of these theories. Our construction is based on the observation that the problem of constructing boundary states for these theories is essentially equivalent to the problem of finding the twisted boundary states of the original theory. These twisted boundary states satisfy two sets of Cardy-like conditions, one coming from the overlaps between the twisted boundary states among themselves, and one from the overlaps between twisted boundary states and the usual Cardy states. These two Cardy conditions combine to give a rather powerful constraint on the twisted boundary states, that will allow us to make a very suggestive proposal. We are also able to show that the NIM-rep entries it defines are always integers, and we have checked explicitly for numerous examples, that these integers are in fact non-negative.

From a mathematical point of view, the WZW models are particularly interesting since many of its quantities can be given a natural algebraic interpretation in terms of the associated affine untwisted Kac-Moody algebra $\mathfrak{g}$. For instance, the highest weight representations of the symmetry algebra $\mathcal{A}$ are labelled by the integrable highest weights $\lambda \in P_{+}$ of $\mathfrak{g}$. The fusion rules $N_{\lambda \mu}^{\nu}$ can be interpreted as Weyl-folded tensor product coefficients

\footnotetext{
* In the diagonal modular invariant, left- and right-moving representations occur in conjugate pairs. See section 2 for a more detailed definition.
} 
of the underlying finite-dimensional algebra $\overline{\mathfrak{g}}$. And the matrix $S$ which diagonalises the fusion rules describes the $\tau \mapsto-1 / \tau$ transformation of the characters $\chi_{\lambda}(\tau)$ of $\mathfrak{g}$.

It is therefore natural to ask three questions about the NIM-reps in WZW models. Namely, find algebraic interpretations for

(a) the boundary state labels $x \in \mathcal{B}$, that is to say the rows and columns of the NIM-reps $\mathcal{N}_{\lambda}$ (in the type III subfactor language [7] these are the $M-N$ sectors);

(b) the NIM-rep coefficients themselves; and

(c) the matrix $\psi$ diagonalising the matrices $\mathcal{N}_{\lambda}$, and expressing the boundary states as linear combinations of the Ishibashi states.

In this paper we suggest answers for the NIM-reps that arise as described above, and that are therefore associated to the symmetries $\omega$ of the (unextended) Dynkin diagram of $\overline{\mathfrak{g}}$. In particular,

(a) the $x \in \mathcal{B}$ are integrable highest weights for the twisted affine algebra $\mathfrak{g}^{\omega}$, or equivalently the highest weights of $\omega$-twisted representations of $\mathfrak{g}$;

(b) the $\mathcal{N}_{\lambda x}^{y}$ are the coefficients of the fusion of the $\omega$-twisted $\mathfrak{g}$-representation $x$ with the untwisted $\mathfrak{g}$-representation $\lambda$; and

(c) $\psi$ is the matrix $S$ describing how the characters of the twisted algebra $\mathfrak{g}^{\omega}$ transform under $\tau \mapsto-1 / \tau$.

The exponents $\mu \in \mathcal{E}$ can also be interpreted as the highest weights of another twisted algebra, the so-called orbit algebra $\check{\mathfrak{g}}$. Just as the symmetries of the (extended) Dynkin diagram of $\mathfrak{g}$ give rise to simple currents and thus to symmetries and gradings of the fusion coefficients, we will find that the symmetries of the Dynkin diagrams of $\mathfrak{g}^{\omega}$ and $\check{\mathfrak{g}}$ give rise to symmetries and gradings of the NIM-rep coefficients.

We also interpret our answers to (a), (b) and (c) in terms of data for certain affine untwisted algebras; in particular we can express our NIM-rep coefficients using ordinary fusion coefficients, proving the integrality of our NIM-reps.

Answers to these questions, which appear to be similar to ours (although they were obtained by independent arguments), appear in work by Fuchs, Schweigert, and collaborators (see in particular [9,10]). However, we feel that the fundamental simplicity of the above algebraic descriptions, and the fact that one can be completely explicit, is rather elusive in their work. In particular, their description is in terms of an orbifold construction, 
and this hides the simple underlying structure we exhibit in our paper. They have also not yet shown that their conjectured NIM-reps are in fact integral.

Given this algebraic description of the NIM-reps, it seems plausible that our construction may generalise further to symmetries that are only symmetries of the extended Dynkin diagram (and that therefore give rise to simple current modular invariants). This is illustrated in section 6 where we construct the NIM-reps for the simple current modular invariants of $\operatorname{su}(p)$ with $p \geq 2$ prime (for $p=2$ we ignore the easy case $k / 2$ odd). As long as $p^{2}$ does not divide $k+p$ we can furthermore prove that the NIM-rep entries are indeed non-negative integers. A more complete description of these 'simple current' NIM-reps and their non-negativity and integrality will be given elsewhere. These simple current NIMreps have been addressed in [11] in considerable generality, although in our opinion their description is much less explicit than our section 6 . They also provide an argument for the integrality and non-negativity of the NIM-rep coefficients, but it depends on the validity of their conjectured formula for the fusion coefficients of the chiral algebra extended by simple currents, and this conjecture has not yet to our knowledge been rigorously established. For recent work on a completely different approach to proving this integrality and non-negativity, see [12].

Surprisingly little is known about explicit NIM-reps, even for the WZW models. The known NIM-reps include at least one corresponding to each modular invariant of su(2) [13] and $\mathrm{su}(3)$ [13, 4,8,9, 3] — see [3] for the lists; at least one NIM-rep for each known modular invariant of $\mathrm{su}(4)$ [14, 7, 8, 9, 11]; and a few NIM-reps for $\operatorname{su}(5)$ and $G_{2}$ [13, 14, 7, 9, 11]. The list of NIM-reps for $\mathrm{su}(2)$ is complete [13]; the lists of 'fully realised' NIM-reps for su(3) and $\mathrm{su}(4)$ have also been claimed to be complete [8], although the arguments have not yet appeared in print. On the other hand, it is known that the lists of all NIM-reps (without assuming them to be 'physical' or 'fully realised') are incomplete for all algebras other than $\mathrm{su}(2)$. As mentioned before, NIM-reps associated to conjugation automorphisms and simple currents are constructed for instance in [9,11]; however their formulae are far more complicated and less explicit than what is done here and in other papers quoted above. In the type III subfactor framework, NIM-reps compatible with the modular invariants corresponding to simple currents [7] and conformal embeddings [15],7], were shown to exist for $\mathrm{su}(n)$, and thus these NIM-reps are in principle computable from the subfactor machinery [16]7], although this computation is not entirely straightforward and has not been performed. The diagonalising matrix $\psi$, however, is at present inaccessible from the subfactor approach. 
While this paper was being completed, [17] appeared in which the NIM-reps corresponding to charge conjugation $C$ for $\operatorname{su}(n)$ are obtained, using different methods than ours. (In particular, their analysis does not involve twisted affine algebras.) Their NIMreps seem to be consistent with ours, although their diagonalising $\psi$-matrices differ from ours by some (immaterial) signs, and their boundary state labels are different (e.g. for $\mathrm{su}(5)$ level 2 , their boundary states $\left(m_{1}, m_{2}\right)=(1,1),(1,3)$ and $(2,1)$ correspond to our labels $\left(a_{1}, a_{2}\right)=(0,1),(0,0)$ and $(1,0)$, respectively). After completing this paper we were also made aware of the paper [18] whose results have some overlap with our section 3.

The paper is organised as follows. In section 2 we describe our conventions and some background material. Our construction of the NIM-reps is motivated in section 3 where we explain how it can be understood in terms of constructing twisted boundary conditions for the original diagonal theory. In section 4 the NIM-reps are explicitly described, and their relation to ordinary fusion matrices is exhibited, thereby proving the integrality of the NIM-rep coefficients. Details of the underlying calculations are given in section 5 . In section 6 we describe the NIM-reps for some simple current modular invariants, and prove that, under suitable conditions, their entries are indeed non-negative integers. Finally, section 7 contains some conclusions. We have included an appendix in which the graphs of some of the NIM-reps are explicitly given for small rank and level.

\section{Conventions and background material}

We will be interested here in the rational conformal field theory associated to an affine untwisted Kac-Moody algebra $\mathfrak{g}$ at positive integer level $k$. Let us denote by $P_{+}=P_{+}^{k}(\overline{\mathfrak{g}})$ the set of all (finitely many) integrable level $k$ highest weight representations of $\mathfrak{g}$. The

corresponding highest weights can be written as $\lambda=\sum_{i=0}^{n} \lambda_{i} \Lambda_{i}=\left(\lambda_{0} ; \lambda_{1}, \ldots, \lambda_{n}\right)$, where the non-negative integers $\lambda_{i}$ are the Dynkin labels of $\lambda$, and the $\Lambda_{i}$ are the fundamental weights of $\mathfrak{g}$. For instance, the vacuum representation corresponds to the weight $k \Lambda_{0}$.

The characters $\chi_{\lambda}(\tau), \lambda \in P_{+}$, of a rational conformal field theory carry a representation of the modular group [19], and in particular

$$
\chi_{\lambda}(-1 / \tau)=\sum_{\mu \in P_{+}} S_{\mu \lambda} \chi_{\mu}(\tau)
$$


where $S$ is a unitary symmetric matrix. By the Verlinde formula [20] the $S$-matrix determines the fusion rule coefficients of the theory as

$$
N_{\lambda \mu}^{\nu}=\sum_{\kappa \in P_{+}} \frac{S_{\lambda \kappa} S_{\mu \kappa} S_{\nu \kappa}^{*}}{S_{0 \kappa}}
$$

Charge conjugation $C$ is a permutation of $P_{+}$, sending the weight $\lambda$ to the weight $\lambda^{c}$ contragredient to it; it is given by $C=S^{2}$, and obeys $N_{\lambda \mu}^{0}=\delta_{\mu, C \lambda}$. $C$ always corresponds to a symmetry of the unextended Dynkin diagram (although this symmetry may be trivial).

Another permutation of $P_{+}$which is of fundamental importance in the theory, is due to the simple currents (see for example [21,22]). These are the weights $J \in P_{+}$with quantum dimension $\frac{S_{J 0}}{S_{00}}=1$; their fusion rules $N_{J, \mu}^{\nu}=\delta_{\nu, J \mu}$ define a permutation $\mu \mapsto J \mu$. This permutation obeys

$$
S_{\lambda J \mu}=e^{2 \pi \mathrm{i} Q(\lambda)} S_{\lambda \mu}
$$

for a rational phase $Q(\lambda)$. For all WZW theories except $\widehat{E}_{8}$ level 2, the simple currents correspond to symmetries of the extended Dynkin diagram. Simple currents give rise to symmetries and gradings of fusion coefficients

$$
\begin{gathered}
N_{J \lambda, J^{\prime} \mu}^{J J^{\prime} \nu}=N_{\lambda \mu}^{\nu} \\
N_{\lambda \mu}^{\nu} \neq 0 \Longrightarrow Q(\lambda)+Q(\mu) \equiv Q(\nu)(\bmod 1) .
\end{gathered}
$$

We will encounter simple currents in our formulae in section 4 for the NIM-rep coefficients, and more explicitly in section 6 .

We shall only consider conformal field theories for which the left- and right-moving chiral algebra is the same, namely the untwisted Kac-Moody algebra $\mathfrak{g}$. The space of states of the full conformal field theory can then be decomposed into representations of two copies of $\mathfrak{g}$, and thus can be written as

$$
\mathcal{H}=\bigoplus_{\lambda, \mu \in P_{+}} M_{\lambda \mu} \mathcal{H}_{\lambda} \otimes \overline{\mathcal{H}_{\mu}^{*}}
$$

where $M_{\lambda \mu}$ are non-negative integers that describe the multiplicity with which the various tensor products of representations appear in $\mathcal{H}$. We shall call the theory defined by $M=I$ the 'diagonal' theory; from the point of view of constructing boundary states, this is the simplest case (as we shall see momentarily). We shall mainly be interested in theories 
that can be defined on the torus; this requires, in particular, that the partition function corresponding to (2.5),

$$
Z(\tau)=\sum_{\lambda, \mu \in P_{+}} M_{\lambda \mu} \chi_{\lambda}(\tau) \chi_{\mu}(\tau)^{*}
$$

is modular invariant. The easiest examples of modular invariants are $M=I$, and $M=C$, where $C$ denotes charge conjugation. More generally, any symmetry of the unextended Dynkin diagram defines a modular invariant; on the other hand, symmetries of the extended Dynkin diagram (i.e. simple currents) may or may not yield a modular invariant for a given fixed level $k$ (for example $k$ must be even for $\mathrm{su}(2)$ ). The symmetries of the unextended Dynkin diagram are often called conjugations.

For a given modular invariant $M$, we call the exponents of $M$ the diagonal terms $M_{\lambda \lambda}$, or more precisely, the 'multi-set' $\mathcal{E}_{M}$ in which $M_{\lambda \lambda}$ copies of primary field $\lambda$ appears, for each $\lambda \in P_{+}$.

We shall be interested in constructing boundary states for these conformal field theories. A boundary state $\| a\rangle\rangle$ is a coherent state in the full conformal field theory. In the simplest case where the boundary preserves the full affine algebra (that is generated by $\left.J_{m}^{b}\right)$ it is characterised by the 'gluing condition'

$$
\left.\left.\left(J_{m}^{b}+\bar{J}_{-m}^{b}\right) \| a\right\rangle\right\rangle=0 \quad \text { for all } b \text { and } m \in \mathbb{Z} .
$$

Since the modes that appear in (2.7) map each $\mathcal{H}_{\lambda} \otimes \overline{\mathcal{H}_{\mu}^{*}}$ into itself, we can solve the gluing condition separately for each summand in (2.5). We can find a non-trivial solution provided that $\mathcal{H}_{\lambda}$ is isomorphic to $\mathcal{H}_{\mu}$, i.e. for each $\lambda \in \mathcal{E}_{M}$. For each such $\lambda$, the relevant coherent state $|\lambda\rangle\rangle$ is then unique up to normalisation, and is called the Ishibashi state. We shall always (partially) fix the normalisation of the Ishibashi states so that

$$
\left\langle\left\langle\lambda\left|q^{\frac{1}{2}\left(L_{0}+\bar{L}_{0}-\frac{c}{12}\right)}\right| \mu\right\rangle\right\rangle=\delta_{\lambda \mu} \chi_{\lambda}(\tau), \quad q=e^{2 \pi i \tau}
$$

Every boundary state can then be written as a linear combination of Ishibashi states,

$$
\left.\| a\rangle\rangle=\sum_{\mu \in \mathcal{E}_{M}} \frac{\psi_{a \mu}}{\sqrt{S_{0 \mu}}}|\mu\rangle\right\rangle,
$$

where we have introduced the factor involving $S_{0 \mu}$ for future convenience. Given the above normalisation of the Ishibashi states, the boundary states are determined in terms of the 
matrix $\psi_{a \mu}{ }^{\dagger}$. In general we expect to be able to find as many boundary states as there are Ishibashi states, i.e. that $\psi$ is a square matrix. Furthermore, the completeness argument of [2] suggests that $\psi$ should be unitary.

Not every linear combination (2.9) defines an actual boundary state. The allowed boundary states have to satisfy a number of consistency conditions, the most important of which is the so-called Cardy condition [1] which can be understood as follow. The 'overlap' between two boundary states can be calculated from (2.9) as

$$
\left\langle\left\langle a\left\|q^{\frac{1}{2}\left(L_{0}+\bar{L}_{0}-\frac{c}{12}\right)}\right\| b\right\rangle\right\rangle=\sum_{\mu \in \mathcal{E}_{M}} \frac{\psi_{a \mu}^{*} \psi_{b \mu}}{S_{0 \mu}} \chi_{\mu}(\tau)
$$

Upon the modular transformation $\tau \mapsto-1 / \tau$, this amplitude must then be expressible in terms of a non-negative integer combination of characters, i.e.

$$
\sum_{\mu \in \mathcal{E}_{M}} \frac{\psi_{a \mu}^{*} \psi_{b \mu}}{S_{0 \mu}} \chi_{\mu}(-1 / \tau)=\sum_{\mu \in \mathcal{E}_{M}} \sum_{\lambda \in P_{+}} \frac{\psi_{a \mu}^{*} \psi_{b \mu}}{S_{0 \mu}} S_{\lambda \mu} \chi_{\lambda}(\tau)=: \sum_{\lambda \in P_{+}} \mathcal{N}_{\lambda b}^{a} \chi_{\lambda}(\tau)
$$

where $\mathcal{N}_{\lambda b}^{a}$, defined by

$$
\mathcal{N}_{\lambda b}^{a}=\sum_{\mu \in \mathcal{E}_{M}} \frac{\psi_{a \mu}^{*} S_{\lambda \mu} \psi_{b \mu}}{S_{0 \mu}}
$$

must be a matrix of non-negative integers. The collection of these matrices is usually called a NIM-rep. More abstractly, we define a NIM-rep $\mathcal{N}$ to be an assignment of a matrix $\mathcal{N}_{\lambda}$, with non-negative integer entries, to each $\lambda \in P_{+}$such that $\mathcal{N}$ forms a representation of the fusion ring

$$
\mathcal{N}_{\lambda} \mathcal{N}_{\mu}=\sum_{\nu \in P_{+}} N_{\lambda \mu}^{\nu} \mathcal{N}_{\nu}
$$

for all primaries $\lambda, \mu, \nu \in P_{+}$. Furthermore we require that

$$
\begin{aligned}
\mathcal{N}_{0} & =I \\
\mathcal{N}_{C \lambda} & =\mathcal{N}_{\lambda}^{t} \quad \lambda \in P_{+} .
\end{aligned}
$$

$\dagger$ Actually, (2.8) only determines the normalisation of the Ishibashi state up to an arbitrary phase. Furthermore, if some $\mu$ appears with non-trivial multiplicity $n$ in $\mathcal{E}_{M}$, we can redefine the Ishibashi states by a transformation in $U(n)$. Unless these choices are specified further, there is therefore an ambiguity in the definition of $\psi$. This accounts for the difference in our formulae for $\psi$ from those in [17]. 
If the NIM-rep arises as in the above construction of boundary states with $\psi$ being unitary, it is easy to see that (2.13) and (2.14) follow from (2.12). The easiest example of a NIMrep are the fusion matrices themselves since the assignment $\lambda \mapsto N_{\lambda}$ clearly satisfies both (2.13) and (2.14).

The matrices $\mathcal{N}_{\lambda}$ of any NIM-rep can always be simultaneously diagonalised, by a unitary matrix $\widehat{\psi}$, in such a way that

$$
\left(\mathcal{N}_{\lambda}\right)_{x y}=\mathcal{N}_{\lambda x}^{y}=\sum_{\mu \in \mathcal{E}} \frac{\widehat{\psi}_{x \mu} S_{\lambda \mu} \widehat{\psi}_{y \mu}^{*}}{S_{0 \mu}} .
$$

The sum will be over some 'multi-subset' $\mathcal{E}=\mathcal{E}(\mathcal{N})$ of $P_{+}$, i.e. each element of $P_{+}$will come with a multiplicity, possibly zero. This multi-set $\mathcal{E}$ is called the exponents of the NIM-rep $\mathcal{N}$. The eigenvalues and simultaneous eigenvectors (i.e. the columns of $\widehat{\psi}$ ) of the matrices $\mathcal{N}_{\lambda}$ are parametrised naturally by the exponents $\mu \in \mathcal{E}(\mathcal{N})$. The entries of these eigenvectors, i.e. the rows of $\widehat{\psi}$, are parametrised by $x \in \mathcal{B}$; the same labels also run through the rows and columns of the matrices $\mathcal{N}_{\lambda}$.

As we shall see later on, some of the NIM-reps that we shall discuss in this paper have an analogue of a simple current symmetry (compare (2.4)). By this we mean permutations $J^{B}$ of $\mathcal{B}$ and $J^{E}$ of $\mathcal{E}$, such that

$$
\begin{aligned}
& \widehat{\psi}_{a J^{E} \mu}=e^{2 \pi \mathrm{i} Q^{E}(a)} \widehat{\psi}_{a \mu} \\
& \widehat{\psi}_{J^{B} a \mu}=e^{2 \pi \mathrm{i} Q^{B}(\mu)} \widehat{\psi}_{a \mu} \\
& \mathcal{N}_{\lambda, J^{B} a}^{J^{B} b}=\mathcal{N}_{\lambda a}^{b} \\
& \mathcal{N}_{\lambda a}^{b} \neq 0 \Longrightarrow Q^{E}(\lambda)+Q^{E}(a) \equiv Q^{E}(b)(\bmod 1),
\end{aligned}
$$

for appropriate phases $Q^{B}$ and $Q^{E}$.

Any modular invariant (boundary) conformal field theory has both a modular invariant $M$ and a NIM-rep $\mathcal{N}$. We say that the set of boundary states is complete, if $M$ and $\mathcal{N}$ are compatible in the sense that their exponents agree (including multiplicities): $\mathcal{E}_{M}=$ $\mathcal{E}(\mathcal{N})$. If this is the case we can then also identify the matrices $\psi=\widehat{\psi}$; this is to say, the diagonalising matrix $\widehat{\psi}$ of the abstract NIM-rep has then an interpretation in terms of the boundary states of the conformal field theory.

One example where this identification has been understood is the diagonal modular invariant theory $M=I$ for which $\mathcal{E}_{M}=P_{+}$. The boundary states of this theory have 
been constructed in [1]; they are labelled by elements in $P_{+}$and defined by $\psi=S$. The corresponding NIM-rep is then the fusion matrix, as follows from (2.2), which again has $\mathcal{E}(\mathcal{N})=P_{+}$.

However, in general it is not known how to construct the NIM-rep that corresponds to the other modular invariants. In this paper we want to solve this problem, in the context of the affine algebras, for those modular invariants that correspond to a symmetry of the unextended Dynkin diagram. We will also get a natural interpretation for the 'boundary state labels' $x \in \mathcal{B}$.

\section{The abstract construction}

As we mentioned in the previous subsection, the boundary states for the diagonal modular invariant are known, and they are given by

$$
\left.\| \lambda\rangle=\sum_{\mu \in P_{+}} \frac{S_{\lambda \mu}}{\sqrt{S_{0 \mu}}}|\mu\rangle\right\rangle .
$$

Suppose $\omega$ is any outer automorphism of $\mathfrak{g}$ that arises from an automorphism of the unextended Dynkin diagram. We want to find the boundary states of the modular invariant based on $\omega$. The exponents $\mathcal{E}_{\omega}$ of the corresponding NIM-rep are precisely the subset of $P_{+}$consisting of those representations that are invariant under the action of $\omega$. The problem of finding the boundary states for the modular invariant based on $\omega$ is therefore essentially equivalent to finding the $\omega$-twisted boundary states for the original diagonal modular invariant theory. A boundary state is $\omega$-twisted if it satisfies the twisted gluing condition

$$
\left.\left.\left(J_{m}^{b}+\omega\left(\bar{J}_{-m}^{b}\right)\right) \| a\right\rangle\right\rangle^{\omega}=0 \quad \text { for all } b \text { and } m \in \mathbb{Z} .
$$

Twisted boundary states have been discussed before in [23]. We shall propose a simple description for these boundary states (and their corresponding NIM-rep) below.

Every twisted boundary state can be written as a linear superposition of the $\omega$-twisted Ishibashi states $|\mu\rangle\rangle^{\omega}$, that can be defined for each $\mu \in \mathcal{E}_{\omega}$, i.e. for each $\mu \in P_{+}$that is invariant under the induced action of $\omega, \omega^{*}(\mu)=\mu$. Again, the Ishibashi states are uniquely determined by this condition up to normalisation, which we (partially) fix by demanding that

$$
{ }^{\omega}\left\langle\left\langle\lambda\left|q^{\frac{1}{2}\left(L_{0}+\bar{L}_{0}-\frac{c}{12}\right)}\right| \mu\right\rangle\right\rangle^{\omega}=\delta_{\lambda \mu} \chi_{\lambda}(\tau) .
$$


Let us expand the $\omega$-twisted boundary states as

$$
\left.\| x\rangle\rangle^{\omega}=\sum_{\mu \in \mathcal{E}_{\omega}} \frac{\psi_{x \mu}}{\sqrt{S_{0 \mu}}}|\mu\rangle\right\rangle^{\omega}
$$

where $x$ is, at this stage, an abstract label for the different twisted boundary states. The NIM-rep that is associated to the $\omega$-twisted boundary states is then simply given by

$$
\mathcal{N}_{\lambda x}^{y}=\sum_{\mu \in \mathcal{E}_{\omega}} \frac{\psi_{y \mu}^{*} S_{\lambda \mu} \psi_{x \mu}}{S_{0 \mu}}
$$

as follows from the same calculation that led to (2.12).

Now we come to describe the main idea of our construction. The advantage of considering the problem in the context of the diagonal modular invariant theory (rather than as the problem of finding the boundary states corresponding to a different theory) is that we do not only have the Cardy condition that requires that the boundary states (3.4) among themselves lead to non-negative integers as in (3.5). If the twisted boundary states (3.4) are consistent, they must also satisfy an appropriate Cardy condition involving the overlap between one of the twisted boundary states, and one of the original Cardy states (3.1). As we shall explain momentarily, this constraint turns out to be very powerful, and will allow us to make a very suggestive proposal for $\psi$.

In order to analyse this constraint we have to determine the overlap between the boundary state $\| \lambda\rangle\rangle$ and the boundary state $\| y\rangle\rangle^{\omega}$. Given our expansion in terms of the relevant Ishibashi states this can be easily done, and we find

$$
{ }^{\omega}\left\langle\left\langle y\left\|q^{\frac{1}{2}\left(L_{0}+\bar{L}_{0}-\frac{c}{12}\right)}\right\| \lambda\right\rangle\right\rangle=\sum_{\mu \in \mathcal{E}_{\omega}} \frac{\psi_{y \mu}^{*} S_{\lambda \mu}}{S_{0 \mu}} \chi_{\mu}^{(\omega)}(\tau),
$$

where $\chi_{\mu}^{(\omega)}(\tau)$ is the twining character in the representation $\mu$, i.e.

$$
\chi_{\mu}^{(\omega)}(\tau)=\operatorname{Tr}_{\mathcal{H}_{\mu}}\left(\tau_{\omega} q^{L_{0}-\frac{c}{24}}\right) .
$$

Here $\tau_{\omega}$ is the induced action of $\omega$ on $\mathcal{H}_{\mu}$.

We can now use the fact that the above twining character agrees precisely with the ordinary character of the so-called orbit Lie algebra $\check{\mathfrak{g}}$ [24]. (Explicit descriptions for these Lie algebras are given in table (2.24) of [24]; see also the translation of their notation to the notation of Kac 25] on page 12.) 
In order to relate the 'closed string result' (3.6) to the 'open string picture' we have to apply again a modular transformation, as before in 2.11). In the present context this involves the characters of $\check{\mathfrak{g}}$ that do not transform into one another under the $S$ modular transformation. Instead, the characters of $\mathfrak{\mathfrak { g }}$ transform into linear combinations of characters of the twisted Lie algebra $\mathfrak{g}^{\omega}$. Let us label the representations of $\mathfrak{g}^{\omega}$ by $\hat{l}$. Then the relevant formula is

$$
\chi_{\mu}^{(\omega)}(-1 / \tau)=\sum_{\hat{l}} \hat{S}_{\hat{l} \mu} \chi_{\hat{l}}(\tau)
$$

where $\hat{S}$ is the corresponding (unitary) $S$-matrix that is given in Thms.13.8, 13.9 of [25] (except that for reasons of convenience we have chosen ours to be the transpose of Kac's). Every (ordinary) representation of the twisted algebra $\mathfrak{g}^{\omega}$ is, by definition, the same as a $\omega$-twisted representation of the original algebra $\mathfrak{g}$. Thus $\hat{l}$ labels equally the $\omega$-twisted representations of $\mathfrak{g}$ (see for example [26] for an introduction into these matters).

Putting all of this together, we can therefore write the overlap (3.6) in the 'open string picture' as

$$
\sum_{\mu \in \mathcal{E}_{\omega}} \sum_{\hat{l}} \frac{\psi_{y \mu}^{*} S_{\lambda \mu} \hat{S}_{\hat{l}_{\mu}}}{S_{0 \mu}} \chi_{\hat{l}}(\tau) .
$$

The relevant 'Cardy' condition is then that

$$
\mathcal{N}_{\hat{l} \lambda}^{y}=\sum_{\mu \in \mathcal{E}_{\omega}} \frac{\psi_{y \mu}^{*} S_{\lambda \mu} \hat{S}_{\hat{l} \mu}}{S_{0 \mu}}
$$

defines non-negative integers.

Next we want to make a suggestive proposal for how to solve the two 'Cardy' conditions (3.5) and (3.10). The NIM-rep we are looking for has dimension $\left|\mathcal{E}_{\omega}\right|$; because $\hat{S}$ is an invertible matrix, this equals the number of $\omega$-twisted representations of the conformal field theory. Now we can always construct $a$ NIM-rep of this dimension by taking $\mathcal{B}$ to label the different $\omega$-twisted (irreducible highest weight) representations, and by taking $\mathcal{N}_{\lambda x}^{y}$ to be the twisted fusion rules that describe the fusion of the untwisted representation $\lambda$ with the twisted representation $x$, leading to twisted representations described by $y$. (The fusion of twisted representations is for example discussed in [27].) These twisted fusion rules automatically define a NIM-rep since fusion is associative.

This suggests therefore that the label $x$ that describes the twisted boundary states can be identified with the label for the $\omega$-twisted representations. Furthermore, the two 
sets of integers (3.5) and (3.10) should both simply be the twisted fusion rules. ${ }^{\ddagger}$ Since these fusion rules are symmetric we therefore have, writing $\hat{l}=x$,

$$
\mathcal{N}_{x \lambda}^{y}=\mathcal{N}_{\lambda x}^{y}
$$

Comparing (3.5) and (3.10) this then implies that

$$
\psi_{x \mu}=\hat{S}_{x \mu}
$$

Thus we propose that the boundary states of the NIM-rep associated to $\omega$ are labelled by the $\omega$-twisted representations, and the NIM-rep itself is precisely described by the twisted fusion rules. The matrix diagonalising the matrices $\mathcal{N}_{\lambda}$ is the modular matrix $\hat{S}$.

Returning to the original problem of finding the boundary states for the conjugation modular invariant, we can now write down a complete set of boundary states, all of which satisfy the gluing condition (2.7). As above, these boundary states are labelled by the $\omega$-twisted representations of $\mathfrak{g}$, and they are given as

$$
\left.\| x\rangle\rangle=\sum_{\mu \in \mathcal{E}_{\omega}} \frac{\hat{S}_{x \mu}}{\sqrt{S_{0 \mu}}}|\mu\rangle\right\rangle,
$$

where $|\mu\rangle\rangle$ are the standard Ishibashi states that exist for $\mu \in \mathcal{E}_{\omega}$. The Cardy condition for these boundary states is now precisely the condition that (3.5) are non-negative integers.

Finally, we should mention that the above arguments imply that we have a generalised Verlinde formula

$$
\mathcal{N}_{x \lambda}^{y}=\sum_{\mu \in \mathcal{E}_{\omega}} \frac{\hat{S}_{y \mu}^{*} S_{\lambda \mu} \hat{S}_{x \mu}}{S_{0 \mu}}
$$

that describes the twisted fusion rules in terms of the $S$-matrices $S_{\lambda \mu}$ and $\hat{S}_{x \mu}$.

\subsection{Generalised fusion algebras}

It is natural to think of our construction in terms of some generalised fusion algebra that combines untwisted and twisted representations. Let us concentrate on the case where

$¥$ After this paper was completed we learned that the possibility of this interpretation in terms of twisted fusion rules was made in passing in 10 . 
the twist is of order two in the following. The fusion matrix corresponding to an untwisted representation $\lambda$ then takes the form

$$
N_{\lambda}^{\text {full }}=\left(\begin{array}{cc}
N_{\lambda \mu}^{\nu} & 0 \\
0 & \mathcal{N}_{\lambda x}^{y}
\end{array}\right)
$$

while the fusion matrix for a twisted representation $x$ is

$$
N_{x}^{\text {full }}=\left(\begin{array}{cc}
0 & \mathcal{N}_{x \mu}^{y} \\
\mathcal{N}_{x y}^{\mu} & 0
\end{array}\right)
$$

In both cases we have written the matrices in blocks corresponding to untwisted $(\lambda, \mu, \nu \in$ $\left.P_{+}\right)$and twisted $(x, y \in \mathcal{B})$ representations. These fusion rules define a consistent fusion algebra in the sense of [4] (although, unlike the more familiar fusion rings of conformal field theory, it is not self-dual).

The NIM-rep that is defined by combining the untwisted and the twisted boundary states is precisely this generalised fusion algebra. In particular, this implies that the NIMreps corresponding to $M=I$ and the one corresponding to the modular invariant associated to the conjugation $\omega$ combine into some larger algebraic structure. This assumption played an essential role in our 'derivation' above.

This is quite analogous to the manner in which group theorists treat projective representations of a finite group $G$ : they are interpreted as true representations of an extension $H$ of the Schur multiplier $M(G)$ of $G$ by $G$. It would be interesting to try to push this analogy further and see, for example, what the Schur multiplier corresponds to in our case.

Since the algebra defined by the matrices (3.15) and (3.16) defines a fusion algebra in the sense of [4], it possesses a (non-symmetric) $S$-matrix that diagonalises the fusion rules, and recovers the full fusion coefficients $N^{\text {full }}$ via Verlinde's formula. In the present context this $\left(\left|P_{+}\right|+\left|\mathcal{E}_{\omega}\right|\right) \times\left(\left|P_{+}\right|+\left|\mathcal{E}_{\omega}\right|\right) S$-matrix is simply given by

$$
S^{\text {full }}=\left(\begin{array}{cc}
\frac{1}{\sqrt{2}} S_{i \mu} & S_{i j} \\
\pm \frac{1}{\sqrt{2}} \hat{S}_{x \mu} & 0
\end{array}\right)
$$

where the first $2\left|E_{\omega}\right|$ columns are parametrised by $\mu \in \mathcal{E}_{\omega}$ and either choice of sign (so each entry ' $\frac{1}{\sqrt{2}} S_{i \mu}$ ' appears twice), and where the final $\left|P_{+}\right|-\left|\mathcal{E}_{\omega}\right|$ columns are parametrised by the $j \in P_{+} \backslash \mathcal{E}_{\omega}$. The first $\left|P_{+}\right|$rows are parametrised by $i \in P_{+}$, and the last $\left|\mathcal{E}_{\omega}\right|$ rows are parametrised by the $\omega$-twisted representations $x$ of $\mathfrak{g}$. If we introduce the column vectors

$$
S_{\uparrow,(\mu, \pm)}^{\text {full }}=\left(\begin{array}{c}
S_{\uparrow, \mu} \\
\pm \hat{S}_{\uparrow, \mu}
\end{array}\right), \quad S_{\uparrow, j}^{\text {full }}=\left(\begin{array}{c}
S_{\uparrow, j} \\
0
\end{array}\right),
$$


then we can rephrase this as follows: for each $\mu$ and choice of sign, the column vector $S_{\downarrow,(\mu, \pm)}^{\text {full }}$ is an eigenvector of $N_{\lambda}^{\text {full }}\left(\lambda \in P_{+}\right)$with eigenvalue $\frac{S_{\lambda \mu}}{S_{0 \mu}}=S_{\lambda,(\mu, \pm)}^{\text {full }} / S_{0,(\mu, \pm)}^{\text {full }}$, and an eigenvector of $N_{x}^{\text {full }}(x \in \mathcal{B})$ with eigenvalue $\pm \frac{\hat{S}_{x \mu}}{S_{0 \mu}}=S_{x,(\mu, \pm)}^{\text {full }} / S_{0,(\mu, \pm)}^{\text {full }}$. Similarly, the column vector $S_{\downarrow, j}^{\text {full }}$ is an eigenvector of $N_{\lambda}^{\text {full }}\left(\lambda \in P_{+}\right)$with eigenvalue $\frac{S_{\lambda j}}{S_{0 j}}=S_{\lambda j}^{\text {full }} / S_{0 j}^{\text {full }}$, and an eigenvector of $N_{x}^{\text {full }}(x \in \mathcal{B})$ with eigenvalue $0=S_{x j}^{\text {full }} / S_{0 j}^{\text {full }}$.

This generalised fusion algebra also appears in [9] under the name 'classifying algebra'. Their equations (4.5) and (4.7) correspond here to (3.17) and (3.15), (3.16), respectively.

One consequence of (3.17) and Perron-Frobenius theory is that, if the coefficients $\mathcal{N}_{\lambda x}^{y}$ are all to be non-negative, the entries $\hat{S}_{x 0}$ must be of constant sign for all $x \in \mathcal{B}$. We will see in the next section that they are indeed always positive. This can be regarded as a non-trivial test of non-negativity — the only property of our NIM-reps which we cannot prove in general. The property of $\hat{S}_{x 0}$ to be positive has also a physical interpretation since $\psi_{x 0}=\hat{S}_{x 0}$ is proportional to the (positive) boundary entropy of the boundary condition described by $x[28]$.

\subsection{Generalised NIM-reps}

Defect lines (that is, non-local operators attached to non-contractible loops) can be inserted into the partition functions of the torus and cylinder, resulting in two other sets of NIM-rep-like data associated to a conformal field theory [6]. These also appear naturally in the subfactor context (see for example Thm. 4.16 in [16]). In particular, to each pair $\lambda, \lambda^{\prime}$ of weights in $P_{+}$, we should have a matrix $\widetilde{V}_{\lambda \lambda^{\prime}}=\left(\widetilde{V}_{\lambda \lambda^{\prime} ; i}^{j}\right)_{i, j \in \tilde{\mathcal{B}}}$ of non-negative integers, giving a representation of the 'double-fusion algebra'

$$
\widetilde{V}_{\lambda \lambda^{\prime}} \widetilde{V}_{\mu \mu^{\prime}}=\sum_{\nu, \nu^{\prime} \in P_{+}} N_{\lambda \mu}^{\nu} N_{\lambda^{\prime} \mu^{\prime}}^{\nu^{\prime}} \widetilde{V}_{\nu \nu^{\prime}}
$$

and obeying both $\widetilde{V}_{\lambda, C \lambda^{\prime} ; \tilde{1}}^{\tilde{1}}=M_{\lambda \lambda^{\prime}}$ and $\widetilde{V}_{\lambda \lambda^{\prime}}^{t}=\widetilde{V}_{C \lambda, C \lambda^{\prime}}$. Here $M$ is the torus partition function of the theory, $C$ denotes charge conjugation, and $\widetilde{V}^{t}$ is the transpose of $\widetilde{V}$. The label $\tilde{1}$ is a special element of $\tilde{\mathcal{B}}$. These conditions imply that the matrices $\widetilde{V}_{\lambda \lambda^{\prime}}$ can be simultaneously diagonalised by some matrix $\widetilde{\psi}$, and that the eigenvalues of $\widetilde{V}_{\lambda \lambda^{\prime}}$ will be $\frac{S_{\lambda \mu} S_{\lambda^{\prime} \mu^{\prime}}}{S_{0 \mu} S_{0 \mu^{\prime}}}$; its multiplicity is required to be $\left(M_{\mu \mu^{\prime}}\right)^{2}$. The other NIM-rep-like data concerns a representation by non-negative integer matrices $\widetilde{\mathcal{N}}_{i}=\left(\tilde{\mathcal{N}}_{i x}^{y}\right)_{x, y \in \mathcal{B}}, i \in \tilde{\mathcal{B}}$, of a (not necessarily commutative) fusion-like ring built out of the matrix $\widetilde{\psi}$ - see section 4.2 of [6] for details. 
In the case where $M$ is a permutation matrix $M_{\lambda \mu}=\delta_{\mu, \pi \lambda}$ (this is, in particular, the case when $M$ is a conjugation modular invariant, as considered in this section, or the simple current modular invariant considered in section 6.1 below), it is easy to find these matrices $\widetilde{V}_{\lambda \lambda^{\prime}}$ and $\widetilde{\mathcal{N}}_{i}$ : in particular, we can naturally identify $\tilde{\mathcal{B}}$ with $P_{+}$, and put $\widetilde{V}_{\lambda \lambda^{\prime}}=N_{\lambda} N_{\pi \lambda^{\prime}}$ and $\widetilde{\mathcal{N}}_{\lambda}=\mathcal{N}_{\lambda}$. Thus these other NIM-rep-like data can also be constructed for the theories considered in this paper.

\section{Explicit formulae}

In this section we shall collect explicit formulae describing the NIM-reps for the various classes of algebras. The NIM-rep is uniquely determined once we know the exponents $\mathcal{E}(\mathcal{N})$,

the eigenvalues $\frac{S_{\lambda \mu}}{S_{0 \mu}}$ for all weights $\lambda \in P_{+}$and exponents $\mu \in \mathcal{E}(\mathcal{N})$, and the diagonalising matrix $\psi_{x \mu}$. As we have explained above, the matrix $\psi$ can be identified with the modular $S$-matrix of the twisted algebra $\mathfrak{g}^{\omega}$. In principle these $S$-matrices are known [25], but as for the case of the more familiar untwisted $S$-matrices, the usual formulae are rather complicated (involving sums over Weyl groups). In the following we shall therefore give simple expressions for both $S$-matrices, following the ideas of [29]. We shall also explain how the NIM-rep coefficients can be expressed in terms of the usual fusion coefficients; this will make manifest that they are integers, as they must be. We conjecture that they are also non-negative, and have verified this on the computer for several algebras and levels. The arguments behind these calculations are given in the following section, and some fusion graphs are exhibited in the appendix. In the following we shall analyse the different algebras case by case; we shall always use the same numbering of nodes as in [25,30].

\subsection{The A-series}

A level $k$ weight $\lambda \in P_{+}$for the $A$-series, i.e. $\operatorname{su}(n)=A_{n-1}$, looks like $\lambda=\sum_{i=0}^{n-1} \lambda_{i} \Lambda_{i}$ where $\sum \lambda_{i}=k$. Charge conjugation $\omega=C$ for $\operatorname{su}(n)$ takes the weight $\lambda$ to the weight $C \lambda=\lambda_{0} \Lambda_{0}+\sum_{i=1}^{n-1} \lambda_{n-i} \Lambda_{i}$. Charge conjugation is non-trivial for su(n), except for $\mathrm{su}(2)$.

The exponents $\mathcal{E}$ here are the $C$-invariant weights, i.e. those $\mu$ with Dynkin labels $\mu_{n-i}=\mu_{i}$ for $1 \leq i \leq n-1$. All of these appear with multiplicity 1 . The eigenvalues, involving ratios of $S$-matrix elements, can be effectively computed as follows. 
To the weight $\lambda=\sum_{i} \lambda_{i} \Lambda_{i}$ associate orthogonal coordinates $\lambda^{+}[\ell]=n-j+\sum_{i=\ell}^{n-1} \lambda_{i}$ for $\ell=1, \ldots, n,^{\star}$ and write $t^{+}(\lambda)=\frac{n(n-1)}{2}+\sum_{\ell=1}^{n-1} \ell \lambda_{\ell}$. Then

$$
S_{\lambda \mu}=s \exp \left[2 \pi \mathrm{i} \frac{t^{+}(\lambda) t^{+}(\mu)}{(k+n) n}\right] \operatorname{det}\left(\exp \left[-2 \pi \mathrm{i} \frac{\lambda^{+}[i] \mu^{+}[j]}{k+n}\right]\right)_{1 \leq i, j \leq n}
$$

where $s$ is some (for our purposes irrelevant) constant that is given in e.g. [29]. Here 'det' denotes the determinant of the $n \times n$ matrix whose $(i, j)$-th entry is provided.

In order to describe the formula for $\psi$, we need to distinguish two cases depending on the parity of $n$.

\section{The case of $\operatorname{su}(2 n+1)$}

As we know, the exponents, which parametrise the eigenvectors, and label the columns of $\psi$, are the $C$-invariant weights of $\mathfrak{g}=\widehat{\mathrm{su}}(2 n+1)$ level $k$. Alternatively, we can think of them as the level $k$ weights of the orbit Lie algebra $\check{\mathfrak{g}}=A_{2 n}^{(2)}$. In either case, they can be equated with the $(n+1)$-tuples $\mu=\left(\mu_{0} ; \mu_{1}, \ldots, \mu_{n}\right)$, where $\mu_{i} \in \mathbb{Z}_{\geq 0}$ and we have $k=\mu_{0}+2 \mu_{1}+\cdots+2 \mu_{n-1}+2 \mu_{n}$. To each exponent $\mu \in \mathcal{E}$ we associate the coordinates $\mu[i]=n+1-i+\sum_{j=i}^{n} \mu_{j}$ for $i=1, \ldots, n$.

The boundary states, which label the components of each eigenvector, and both the rows and columns of each NIM-rep matrix $\mathcal{N}_{\lambda}=\left(\mathcal{N}_{\lambda a}^{b}\right)$, as well as the rows of $\psi$, are the level $k C$-twisted weights of $\mathfrak{g}=\widehat{\mathrm{su}}(2 n+1)$, or alternatively the level $k$ weights of the twisted Lie algebra $\mathfrak{g}^{\omega}=A_{2 n}^{(2)}$. They can be equated with all $(n+1)$-tuples $\left(a_{0} ; a_{1}, \ldots, a_{n}\right)$ where $k=a_{0}+2 a_{1}+2 a_{2}+\cdots+2 a_{n}$ and $a_{i} \in \mathbb{Z}_{\geq 0}$. To each such $a$ we associate the coordinates $a[i]=n+1-i+\sum_{j=i}^{n} a_{j}$ for $i=1, \ldots, n$.

Then the $\psi$-matrix, that is to say the modular matrix $\hat{S}$ for $A_{2 n}^{(2)}$, is ${ }^{\dagger}$

$$
\psi_{a \mu}=(-1)^{\frac{n(n-1)}{2}} \frac{2^{n}}{(k+2 n+1)^{\frac{n}{2}}} \operatorname{det}\left[\sin \left(\frac{2 \pi a[i] \mu[j]}{k+2 n+1}\right)\right]_{1 \leq i, j \leq n}
$$

We will show in section 5 that the column of $\psi$ corresponding to $\mu^{0}=(k ; 0, \ldots, 0)$, and the row of $\psi$ corresponding to $a^{0}=(k ; 0, \ldots, 0)$ are both strictly positive.

Incidentally, this $\psi$-matrix can be regarded as a symmetric submatrix of the $S$-matrix for $\widehat{B}_{n}$ level $k+2$, provided we identify $a$ and $\mu$ with the appropriate weights $a^{\prime}, \mu^{\prime}$ in

* We shall always use the convention that $\sum_{i=r}^{r-1}=0$.

$\dagger$ This determinant formula for $\hat{S}$ for the case of $A_{2 n}^{(2)}$ first appeared in [24]. They also noted that when $k$ is odd, $\hat{S}$ equals the $S$-matrix for $\widehat{C}_{n}$ level $\frac{k-1}{2}$. 
$P_{+}^{k+2}\left(B_{n}\right)$. More specifically, we identify $\mu \mapsto \mu^{\prime}=\left(\mu_{0}+\mu_{1}+1 ; \mu_{1}, \ldots, \mu_{n-1}, 2 \mu_{n}+1\right)$ and $a \mapsto a^{\prime}=\left(a_{0}+a_{1}+1 ; a_{1}, \ldots, a_{n-1}, 2 a_{n}+1\right) .^{\ddagger}$ With these identifications, the $\psi$-matrix becomes symmetric, and the relation between $\psi$ and the $\widehat{B}_{n}$ level $k+2 S$-matrix $S^{\prime}$ is simply $\psi_{a \mu}=2 S_{a^{\prime} \mu^{\prime}}^{\prime}$

Using this identification, we can express the NIM-rep coefficients $\mathcal{N}_{\lambda a}^{b}$, corresponding via (3.5) to (4.2), in terms of the ordinary fusion coefficients $N^{\prime}$ of $\widehat{B}_{n}$ level $k+2$, and branching rules for $B_{n} \subset \mathrm{su}(2 n+1)$,

$$
\mathcal{N}_{\lambda a}^{b}=\sum_{\gamma^{\prime}} b_{\gamma^{\prime}}^{\lambda}\left(N_{\gamma^{\prime}, a^{\prime}}^{\prime} b^{\prime}-N_{J \gamma^{\prime}, a^{\prime}}^{\prime} b^{\prime}\right) .
$$

Here, $J^{\prime}$ is the simple current of $\widehat{B}_{n}$, which acts on weights $\nu^{\prime}$ by $J \nu^{\prime}=\left(\nu_{1}^{\prime} ; \nu_{0}^{\prime}, \nu_{2}^{\prime}, \ldots, \nu_{n}^{\prime}\right)$. The coefficients $b_{\gamma^{\prime}}^{\lambda}$ describe the branching rules $\oplus_{\gamma^{\prime}} b_{\gamma^{\prime}}^{\lambda}\left(\gamma^{\prime}\right)=(\lambda)$ for the embedding of $B_{n} \subset \mathrm{su}(2 n+1)$; some branching rules for $B_{n} \subset \mathrm{su}(2 n+1)$ are given for instance in [31]. The sum in (4.3) is over all dominant weights $\gamma^{\prime}$ of $B_{n}$; we extend the definition of the fusion coefficient $N_{\gamma^{\prime}, a^{\prime}}^{\prime}{ }^{b^{\prime}}$ to arbitrary dominant weight $\gamma^{\prime}$ in the obvious way (compare the Kac-Walton fusion formula): the fusion coefficient is $\operatorname{det}(w) N_{w \cdot \gamma^{\prime}, a^{\prime}}^{b^{\prime}}$ if there is a unique element $w$ of the affine Weyl group of $\widehat{B}_{n}$ for which $w \cdot \gamma^{\prime}:=w\left(\gamma^{\prime}+\rho\right)-\rho$ lies in $P_{+}^{k+2}\left(B_{n}\right)$, and it is zero if there are more than one such $w^{\prime}$ s (i.e. if $\gamma^{\prime}$ lies on the boundary of the Weyl alcove). Equation (4.3) makes it manifest that our coefficients $\mathcal{N}_{\lambda a}^{b}$ are integers.

When the level $k$ is odd, a simplification occurs in that we can identify the boundary state $a$ with the entire $\widehat{C}_{n}$ level $\frac{k-1}{2}$ weight $\widetilde{a}=\left(\frac{a_{0}-1}{2} ; a_{1}, \ldots, a_{n}\right)$, and similarly for $\mu$. With this, $\psi$ becomes the $\widehat{C}_{n}$ level $\frac{k-1}{2} S$-matrix, and the NIM-rep becomes

$$
\mathcal{N}_{\lambda a}^{b}=\sum_{\tilde{\gamma}} b_{\tilde{\gamma}}^{\lambda} \widetilde{N}_{\tilde{\gamma} \tilde{a}}^{\tilde{b}}
$$

where $\oplus b_{\tilde{\gamma}}^{\lambda}(\tilde{\gamma})=(\lambda)$ are the branching rules for $C_{n} \subset \operatorname{su}(2 n+1)$. As for (4.3), the fusion coefficients $\widetilde{N}_{\tilde{\gamma} \tilde{a}} \tilde{b}$ are defined for $\tilde{\gamma}$ not necessarily in $\tilde{P}_{+}$, in which case they can be negative. Some branching rules for $C_{n} \subset \mathrm{su}(2 n+1)$ are given in [31].

This simplification reveals a hidden symmetry in the NIM-reps at odd level $k$ : the $\widehat{C}_{n}$ simple current $\tilde{J}=: J^{B}$ acts on $a \in \mathcal{B} \cong P_{+}^{\frac{k-1}{2}}\left(C_{n}\right)$ by $J^{B} a=\left(2 a_{n}+1 ; a_{n-1}, \ldots, a_{1}, \frac{a_{0}-1}{2}\right)$ with phase $Q^{B}(\mu)=\frac{1}{2} \sum_{i=1}^{n} i \mu_{i}$, and $\mathcal{N}_{\lambda, J^{B} a}^{J^{B} b}=\mathcal{N}_{\lambda a}^{b}$ (see $\left.(2.16)\right)$.

Some low-rank clarifications are needed in both (4.3) and (4.4). By $\widehat{B}_{1}$ level $k+2$ we mean affine $\mathrm{su}(2)$ at level $2(k+2)$. Also, $\mathrm{su}(2)=\mathrm{so}(3)$ is embedded in $\mathrm{su}(3)$ in two

$\ddagger P_{+}^{k}\left(B_{n}\right)$ consists of the weights $\mu^{\prime}=\left(\mu_{0}^{\prime} ; \mu_{1}^{\prime}, \ldots, \mu_{n}^{\prime}\right)$ for which $\mu_{0}^{\prime}+\mu_{1}^{\prime}+2 \sum_{i=2}^{n-1} \mu_{i}^{\prime}+\mu_{n}^{\prime}=k$. 
different ways; one has branching rule $(2)=(1,0)$ and the other has $(0) \oplus(1)=(1,0)$. By $B_{1} \subset \mathrm{su}(3)$ we mean the former embedding, and by $C_{1} \subset \mathrm{su}(3)$ we mean the latter. Similarly $\operatorname{sp}(4)=\mathrm{so}(5)$ is embedded in $\mathrm{su}(5)$ in two different ways: one with branching rule $(1,0)=(1,0,0,0)$ (using the so(5) Dynkin labelling) and the other with $(1,0) \oplus(0,0)=$ $(1,0,0,0)$ (using the sp(4) Dynkin labelling). By $B_{2} \subset \operatorname{su}(5)$ we mean the former and by $C_{2} \subset \mathrm{su}(5)$ we mean the latter.

\section{The case of $\operatorname{su}(2 n)$}

Next we determine the formula for $\psi$ for $\mathfrak{g}=\widehat{\operatorname{su}}(2 n)$ at level $k$. The twisted algebra $\mathfrak{g}^{\omega}$ here is $\mathfrak{g}^{\omega}=A_{2 n-1}^{(2)}$ (for $n>2$ ), or $D_{3}^{(2)}$ with nodes 1 and 2 interchanged (for $n=2$ ). The rows of $\psi$ are now labelled by $(n+1)$-tuples $a$ with $k=a_{0}+a_{1}+2 a_{2}+\cdots+2 a_{n}$. To each such $a$ we associate the coordinates $a[i]=n+1-i+\sum_{j=i}^{n} a_{j}$ for $i=1, \ldots, n$. The orbit Lie algebra is $\check{\mathfrak{g}}=D_{n+1}^{(2)}$; each exponent $\mu \in \mathcal{E}$ can be regarded as an $(n+1)$ tuple $\mu$, where $k=\mu_{0}+2 \mu_{1}+\cdots+2 \mu_{n-1}+\mu_{n}$. To it we associate the coordinates $\mu[i]=2 n+1-2 i+2 \sum_{j=i}^{n-1} \mu_{j}+\mu_{n}$. Then the $\psi$-matrix (i.e. $\widehat{S}$ for $A_{2 n-1}^{(2)}$ ) is

$$
\psi_{a \mu}=(-1)^{\frac{n^{2}-n}{2}}(k+2 n)^{-\frac{n}{2}} 2^{n-\frac{1}{2}} \operatorname{det}\left[\sin \left(\frac{\pi a[i] \mu[j]}{k+2 n}\right)\right]_{1 \leq i, j \leq n} .
$$

Although $\psi$ is non-symmetric, both the column corresponding to $\mu^{0}=(k ; 0, \ldots, 0)$ and the row corresponding to $a^{0}=(k ; 0, \ldots, 0)$ are strictly positive.

We can interpret the resulting NIM-rep coefficients $\mathcal{N}_{\lambda a}^{b}$ using fusions $N^{\prime}$ and branching coefficients $b_{\gamma^{\prime}}^{\lambda}$ for the semisimple algebra $\operatorname{su}(2) \oplus \cdots \oplus \mathrm{su}(2)$ ( $n$ times) at level $k+2 n-2$

$$
\mathcal{N}_{\lambda a}^{b}=\sum_{d} \sum_{\gamma^{\prime}} \sum_{\pi} \epsilon(\pi) b_{\gamma^{\prime}}^{\lambda} N_{J^{d} \gamma^{\prime}, \pi a^{\prime}}^{b^{\prime}}
$$

This formula will be explained in more detail in section 5 . We can replace the sum over $\pi$ with the determinant of an $n \times n$ matrix made up of various $A_{1}$ fusions. Even so, equation (4.6) is not very practical, but it does make it manifest that our coefficients $\mathcal{N}_{\lambda a}^{b}$ are integers.

The symmetry of the Dynkin diagram of $\check{\mathfrak{g}}=D_{n+1}^{(2)}$ gives rise to a simple current symmetry $J^{E}$ of $\mathcal{E}$, while the symmetry of the Dynkin diagram of $\mathfrak{g}^{\omega}=A_{2 n-1}^{(2)}$ yields a simple current symmetry $J^{B}$ of $\mathcal{B}$ - see (2.16). These will be described in section 5.3. 


\subsection{The D-series}

Next we consider the algebra $\widehat{\operatorname{so}}(2 n)=\widehat{D}_{n}$ at level $k$. A weight $\lambda \in P_{+}$here satisfies $k=\lambda_{0}+\lambda_{1}+\lambda_{n-1}+\lambda_{n}+2 \sum_{i=2}^{n-2} \lambda_{i}$.

As before, we need to compute effectively certain ratios of $S$-matrix elements. In order to do so we associate to the weight $\lambda=\left(\lambda_{0} ; \lambda_{1}, \lambda_{2}, \ldots, \lambda_{n}\right)$ the orthogonal coordinates $\lambda^{+}[\ell]=n-\ell+\sum_{i=\ell}^{n-1} \lambda_{i}+\frac{\lambda_{n}-\lambda_{n-1}}{2}$ for $\ell=1, \ldots, n$. Then

$S_{\lambda \mu}=s\left\{\operatorname{det}\left[\cos \left(2 \pi \frac{\lambda^{+}[i] \mu^{+}[j]}{k+2 n-2}\right)\right]_{1 \leq i, j \leq n}+(-\mathrm{i})^{n} \operatorname{det}\left[\sin \left(2 \pi \frac{\lambda^{+}[i] \mu^{+}[j]}{k+2 n-2}\right)\right]_{1 \leq i, j \leq n}\right\}$,

where $s$ is some irrelevant constant (which is again given in e.g. [29]).

\section{The case of so( $2 n)$ with chirality flip}

The chirality flip $\omega=P$ interchanges the $n$th and $(n-1)$ st Dynkin labels, $\lambda_{n} \leftrightarrow \lambda_{n-1}$. The chirality flip $P$ agrees with charge conjugation $C=S^{2}$ when $n$ is odd, but $C$ is trivial when $n$ is even. The relevant orbit Lie algebra here is $\check{\mathfrak{g}}=A_{2 n-3}^{(2)}$. The exponents are the $P$-invariant $\widehat{D}_{n}$ weights, i.e. the weights of the form $\left(\mu_{0} ; \mu_{1}, \mu_{2}, \ldots, \mu_{n-1}, \mu_{n-1}\right)$ where $k=\mu_{0}+\mu_{1}+2 \mu_{2}+\cdots+2 \mu_{n-1}$. Given any $P$-invariant $D_{n}^{(1)}$ weight $\mu$, define its coordinates by $\mu[i]=n-i+\sum_{j=i}^{n-1} \mu_{j}$, for $i=1, \ldots, n-1$.

The relevant twisted algebra here is $\mathfrak{g}^{\omega}=D_{n}^{(2)}$. The boundary states are all $n$-tuples $\left(a_{0} ; a_{1}, \ldots, a_{n-1}\right)$ of non-negative integers such that $k=a_{0}+2 a_{1}+2 a_{2}+\cdots+2 a_{n-2}+a_{n-1}$. Define coordinates $a[i]=2 n-1-2 i+2 \sum_{j=i}^{n-2} a_{j}+a_{n-1}$ for $i=1, \ldots, n-1$. Then the $\psi$-matrix (i.e. the matrix $\widehat{S}$ of $D_{n}^{(2)}$ ) is given by

$$
\psi_{a \mu}=\left(\frac{1}{k+2 n-2}\right)^{\frac{n-1}{2}} 2^{n-2} \sqrt{2} \mathrm{i}^{n^{2}+n+2} \operatorname{det}\left[\sin \left(\frac{\pi a[i] \mu[j]}{k+2 n-2}\right)\right]_{1 \leq i, j \leq n-1} .
$$

Again $\psi$ is non-symmetric, but both its $\mu^{0}=(k ; 0, \ldots, 0)$ column and $a^{0}=(k ; 0, \ldots, 0)$ row are strictly positive.

The NIM-rep coefficients can be interpreted in terms of fusions $N^{\prime}$ of $\widehat{B}_{n-1}$ at level $k+1$, and branching coefficients $b_{\gamma^{\prime}}^{\lambda}$ of $B_{n-1} \subset \mathrm{so}(2 n)$,

$$
\mathcal{N}_{\lambda a}^{b}=\sum_{\gamma^{\prime}} b_{\gamma^{\prime}}^{\lambda}\left(N_{\gamma^{\prime}, a^{\prime}}^{\prime} b^{\prime}-N_{J \gamma^{\prime}, a^{\prime}}^{\prime} b^{\prime}\right)
$$

This will be explained in section 5.2; as before it demonstrates that our coefficients $\mathcal{N}_{\lambda a}^{b}$ are integers. 
The symmetry of the Dynkin diagram of $\check{\mathfrak{g}}=A_{2 n-1}^{(2)}$ gives rise to a simple current symmetry $J^{E}$ of $\mathcal{E}$, while the symmetry of the Dynkin diagram of $\mathfrak{g}^{\omega}=D_{n}^{(2)}$ yields a simple current symmetry $J^{B}$ of $\mathcal{B}$ - see (2.16). These will be described in section 5.3.

\section{The case of so(8) with triality}

There are 4 other non-trivial conjugations for so(8), but their NIM-reps are determined (see the end of this subsection) once we know the NIM-rep corresponding to 'triality' $\omega=T$. The order-3 triality maps the Dynkin labels $\mu=\left(\mu_{0} ; \mu_{1}, \mu_{2}, \mu_{3}, \mu_{4}\right)$ to $\left(\mu_{0} ; \mu_{4}, \mu_{2}, \mu_{1}, \mu_{3}\right)$. The relevant twisted algebra here is $\mathfrak{\mathfrak { g }}=\mathfrak{g}^{\omega}=D_{4}^{(3)}$. The exponents are the $\widehat{D}_{4}$ weights of the form $\left(\mu_{0} ; \mu_{1}, \mu_{2}, \mu_{1}, \mu_{1}\right)$ where $k=\mu_{0}+3 \mu_{1}+2 \mu_{2}$. Given any such exponent $\mu$, define coordinates $\mu[1]=3 \mu_{1}+\mu_{2}+4, \mu[2]=\mu_{2}+1$.

The boundary states are labelled by all triples $\left(a_{0} ; a_{1}, a_{2}\right)$ of non-negative integers such that $k=a_{0}+2 a_{1}+3 a_{2}$. Define coordinates $a[1]=a_{1}+a_{2}+2, a[2]=a_{2}+1$. Put $\kappa=k+6$. Then the $\psi$-matrix (i.e. the matrix $\widehat{S}$ for $D_{4}^{(3)}$ ) is

$$
\begin{aligned}
\psi_{a \mu}=\frac{2}{\kappa}( & c\left(m m^{\prime}+2 m n^{\prime}+2 n m^{\prime}+n n^{\prime}\right)+c\left(2 m m^{\prime}+m n^{\prime}+n m^{\prime}-n n^{\prime}\right) \\
& +c\left(-m m^{\prime}+m n^{\prime}+n m^{\prime}+2 n n^{\prime}\right)-c\left(2 m m^{\prime}+m n^{\prime}+n m^{\prime}+2 n n^{\prime}\right) \\
& \left.-c\left(m m^{\prime}+2 m n^{\prime}-n m^{\prime}+n n^{\prime}\right)-c\left(m m^{\prime}-m n^{\prime}+2 n m^{\prime}+n n^{\prime}\right)\right),
\end{aligned}
$$

where $c(x)=\cos \left(\frac{2 \pi x}{3 \kappa}\right)$, and we take $m=a[1], n=a[2], m^{\prime}=\mu[1], n^{\prime}=\mu[2]$. Again $\psi$ is non-symmetric, but both its $\mu^{0}=(k ; 0,0,0,0)$ column and $a^{0}=(k ; 0,0,0,0)$ row are strictly positive.

We can find an interpretation of the NIM-rep coefficients $\mathcal{N}$ in terms of fusions $N^{\prime}$ and branching coefficients $b_{\gamma^{\prime}}^{\lambda}$ of $A_{2}$ at level $k+3$

$$
\mathcal{N}_{\lambda a}^{b}=\sum_{i=0}^{2} \sum_{\gamma^{\prime}} b_{\gamma^{\prime}}^{\lambda}\left(N_{J^{\prime i} \gamma^{\prime}, a^{\prime}}^{b^{\prime}}-N_{J^{\prime i} \gamma^{\prime}, C^{\prime} a^{\prime}}^{b^{\prime}}\right) .
$$

This is further explained in section 5. The formula (4.11) implies, in particular, that our NIM-rep is manifestly integral.

We can now give the NIM-reps for the 3 remaining conjugations of so(8). The NIMrep for the conjugation $T^{-1}: \lambda_{1} \rightarrow \lambda_{4} \rightarrow \lambda_{3} \rightarrow \lambda_{1}$ equals that for triality $T$, given above. The NIM-rep for the conjugation $T^{-1} P: \lambda_{1} \leftrightarrow \lambda_{3}$ is given by $\lambda \mapsto \mathcal{N}_{T \lambda}^{P}$, where $\mathcal{N}^{P}$ is the NIM-rep for chirality flip, while that for $T P: \lambda_{1} \leftrightarrow \lambda_{4}$ is $\lambda \mapsto \mathcal{N}_{T^{-1} \lambda}^{P}$. 


\subsection{The algebra $E_{6}$}

The final algebra with a non-trivial conjugation is $E_{6}$. Its level $k$ weights $\lambda$ satisfy $k=$ $\lambda_{0}+\lambda_{1}+2 \lambda_{2}+3 \lambda_{3}+2 \lambda_{4}+\lambda_{5}+2 \lambda_{6}$. The order- 2 charge conjugation $\omega=C$ interchanges the 1st and 5th, and 2nd and 4th, Dynkin labels, and fixes the 0th, 3rd, and 6th. The relevant twisted algebra here is $\check{\mathfrak{g}}=\mathfrak{g}^{\omega}=E_{6}^{(2)}$.

The ratios of $S$-matrix elements can be effectively calculated using the formula of [29]. The exponents are the $C$-invariant $E_{6}$ weights, i.e. the $\mu$ of the form $\left(\mu_{0} ; \mu_{1}, \mu_{2}, \mu_{3}, \mu_{2}, \mu_{1}, \mu_{6}\right)$ where $k=\mu_{0}+2 \mu_{1}+4 \mu_{2}+3 \mu_{3}+2 \mu_{6}$. Define coordinates by $\mu[1]=2 \mu_{1}+3 \mu_{2}+2 \mu_{3}+\mu_{6}+8, \mu[2]=\mu_{2}+\mu_{3}+\mu_{6}+3, \mu[3]=\mu_{2}+\mu_{3}+2$, and $\mu[4]=\mu_{2}+1$.

The boundary states are all quintuples $\left(a_{0} ; a_{1}, a_{2}, a_{3}, a_{4}\right)$ of non-negative integers such that $k=a_{0}+2 a_{1}+3 a_{2}+4 a_{3}+2 a_{4}$. Define coordinates $a[1]=a_{1}+\frac{3}{2} a_{2}+2 a_{3}+a_{4}+\frac{11}{2}$, $a[2]=\frac{1}{2} a_{2}+a_{3}+a_{4}+\frac{5}{2}, a[3]=\frac{1}{2} a_{2}+a_{3}+\frac{3}{2}$, and $a[4]=\frac{1}{2} a_{2}+\frac{1}{2}$. Put $\kappa=k+12$. Then the $\psi$-matrix (i.e. the matrix $\widehat{S}$ for $E_{6}^{(2)}$ ) is

$$
\begin{gathered}
\psi_{a \mu}=\frac{16}{\kappa^{2}}\left\{\operatorname{det}\left[\sin \left(\frac{2 \pi a[i] \mu[j]}{\kappa}\right)\right]_{1 \leq i, j \leq 4}+\operatorname{det}\left[\sin \left(\frac{2 \pi(c . a)[i] \mu[j]}{\kappa}\right)\right]_{1 \leq i, j \leq 4}\right. \\
\left.+\operatorname{det}\left[\sin \left(\frac{2 \pi\left(c^{t} \cdot a\right)[i] \mu[j]}{\kappa}\right)\right]_{1 \leq i, j \leq 4}\right\} .
\end{gathered}
$$

Here, $(c . a)[i]$ denotes the $i$ th coordinate of the matrix product of $c$ with the column vector with entries $a[j]$, and $c^{t}$ means the transpose(=inverse) of $c$, where $c$ is the orthogonal matrix

$$
c=\frac{1}{2}\left(\begin{array}{cccc}
1 & 1 & 1 & -1 \\
1 & 1 & -1 & 1 \\
1 & -1 & 1 & 1 \\
1 & -1 & -1 & -1
\end{array}\right) .
$$

Again, $\psi$ is non-symmetric but the $a^{0}=(k ; 0, \ldots, 0)$ row is strictly positive, and the same appears to hold for the $\mu^{0}=(k ; 0, \ldots, 0)$ column. The positivity of the $a^{0}$ row can be shown in the usual manner, but the arguments to prove the positivity of the $\mu^{0}$ column are much messier; we have therefore only verified this claim on a computer for levels up to $k=100$.

We can interpret our $\widehat{E}_{6}$ NIM-reps using $\widehat{\mathrm{sO}}(8)$ fusion coefficients $N^{\prime}$ and branching coefficients $b_{\gamma^{\prime}}^{\lambda}$

$$
\mathcal{N}_{\lambda a}^{b}=\sum_{J^{\prime}} \sum_{\gamma^{\prime}} \sum_{\pi} b_{\gamma^{\prime}}^{\lambda} \epsilon(\pi) N_{J^{\prime} \gamma^{\prime}, \pi a^{\prime}}^{b^{\prime}}
$$

This sum (4.14) is manifestly an integer. 


\section{Calculations and further elaborations}

In this section we want to provide details of the derivation of the various formulae we gave in the previous section. First we shall explain how we obtained the formulae for $\hat{S}=\psi$. As an example we shall discuss the calculation for the algebra $\overline{\mathfrak{g}}=\mathrm{su}(2 n)$ explicitly.

\subsection{Derivation of the $\psi$-formula for $\overline{\mathfrak{g}}=\mathrm{su}(2 n)$}

From the discussion of section 3, we need to identify the quantities of Thm. 13.9 in [25]. The finite Weyl group of both $\check{\mathfrak{g}}=D_{n+1}^{(2)}$ and $\mathfrak{g}^{\omega}=A_{2 n-1}^{(2)}$ is just the Weyl group of $\operatorname{sp}(2 n)$, so the $\psi$ formula will involve a determinant that will look like the one in the $S$-matrix for $\widehat{\mathrm{sp}}(2 n)$. Furthermore, we need to be able to evaluate inner products $(a \mid \mu)$, or equivalently we need the values of $\left(\Lambda_{i} \mid \Lambda_{j}^{\prime}\right)$ for the fundamental weights $\Lambda_{i}, \Lambda_{j}^{\prime}$ of $\mathfrak{g}^{\omega}$ and $\check{\mathfrak{g}}$, respectively. To do this we use the equation (see page 222 of [25]) that $\left(\alpha_{j}^{\vee} \mid \Lambda_{i}\right)=\delta_{i j}$ for the coroots $\alpha_{j}^{\vee}$ of $\mathfrak{g}^{\omega}$, where the values of $\left(\alpha_{i}^{\vee} \mid \alpha_{j}^{\vee}\right)$ are determined from the Cartan matrix of $A_{2 n-1}^{(2)}$ (see (6.2.1) of [25]). We obtain the realisation $\Lambda_{i}=e_{1}+\cdots+e_{i}$ for all $i=1, \ldots, n$, where $e_{i}$ is an orthonormal basis of $\mathbb{R}^{n}$. We now find $\Lambda_{i}^{\prime}=\Lambda_{i}$ for $i<n$, and $\Lambda_{n}^{\prime}=\frac{1}{2} \Lambda_{n}$, by page 266 of [25]. This implies that we should define the orthogonal coordinates $\mu[i]$ and $a[i]$, as we did in subsection 4.1, and rescale the inner products by 2 .

The overall multiplicative factor is computed as follows. The number of positive roots of the horizontal algebra $C_{n}$ of $\mathfrak{g}^{\omega}$ is $n^{2}$. The lattice $M^{\prime}$ is the lattice spanned by the coroots $\alpha_{i}^{\vee}$, which is the square lattice $\mathbb{Z}^{n}$. The lattice $M$ is the lattice spanned by the roots $\alpha_{i}$, which is the $D_{n}$ root lattice (see page 93 of [25]). The index $\left|M^{\prime} / M\right|$ is $\sqrt{2}$, and the index $\left|M^{*} /\left(k+h^{\vee}\right) M\right|$ is $4(k+2 n)^{n}$. We want to replace $e^{-\mathrm{i} z}-e^{\mathrm{i} z}$ with $-2 \mathrm{i} \sin (z)$, and so we get an additional factor of $2^{n} \mathrm{i}^{-n}$. Collecting all these terms, we recover (4.5).

\subsection{Relation of NIM-reps to ordinary fusion rules}

Now we turn to the derivations of the fusion formulae for our NIM-reps. Consider first the easiest case: $\operatorname{su}(2 n+1)$ when $k$ is odd. Here, we noticed that $\psi_{a \mu}=\widetilde{S}_{\tilde{a} \tilde{\mu}}$, where the tilde denotes quantities for $C_{n}$ level $\frac{k-1}{2}$. The map $\mu \mapsto \tilde{\mu}$ described in section 4.1 is onto all of $P_{+}^{\frac{k-1}{2}}\left(C_{n}\right)$. We know that an $\mathrm{su}(2 n+1)$ character, restricted to the Cartan subalgebra (CSA) of $C_{n} \subset \mathrm{su}(2 n+1)$, equals the sum of $C_{n}$ characters, the sum given by the appropriate branching coefficients $b_{\tilde{\gamma}}^{\lambda}$. Now, the $\operatorname{su}(2 n+1)$ ratio $\frac{S_{\lambda \mu}}{S_{0 \mu}}$ is the $\lambda$-character of $\mathrm{su}(2 n+1)$ evaluated at the element $-2 \pi \mathrm{i} \frac{\mu+\rho}{k+2 n+1}$ in the CSA not only of $\mathrm{su}(2 n+1)$, but in fact of the embedded algebra $C_{n}$, since $C \mu=\mu$. This means that it equals the sum 
$\sum_{\tilde{\gamma}} b_{\tilde{\gamma}}^{\lambda} \widetilde{S}_{\tilde{\gamma} \tilde{\mu}} / \widetilde{S}_{\tilde{0} \tilde{\mu}}$ of $C_{n}$ characters evaluated at that element of the CSA of $C_{n}$. Thus we have succeeded in expressing all $\mathrm{su}(2 n+1)$ quantities in (3.5) in terms of $C_{n}$ quantities, and we obtain (4.4) from [20] and

$$
\mathcal{N}_{\lambda a}^{b}=\sum_{\mu \in \mathcal{E}} \frac{\psi_{b \mu}^{*} S_{\lambda \mu} \psi_{a \mu}}{S_{0 \mu}}=\sum_{\tilde{\gamma}} b_{\tilde{\gamma}}^{\lambda} \sum_{\tilde{\mu} \in \tilde{P}_{+}} \frac{\tilde{S}_{\tilde{b} \tilde{\mu}}^{*} \tilde{S}_{\tilde{\gamma} \tilde{\mu}} \tilde{S}_{\tilde{a} \tilde{\mu}}}{\tilde{S}_{\tilde{0} \tilde{\mu}}}
$$

where here (as throughout this paper) we extend the definition of fusion coefficients and $S$-entries to arbitrary dominant weights, as explained after (4.3).

Next we want to verify (4.3). The only modification that arises relative to the previous case is that the set of all $\mu^{\prime}$ is only a subset of all weights in $P_{+}^{\prime}:=P_{+}^{k+2}\left(B_{n}\right)$, namely all those weights with $\mu_{n}^{\prime}$ odd and $\mu_{0}^{\prime}>\mu_{1}^{\prime}$. In order to recover the $B_{n}$ fusions from (3.5), we need to extend the sum from all these $\mu^{\prime}$ associated to $\mu \in \mathcal{E}$, to all weights $\mu^{\prime} \in P_{+}^{\prime}$. This is where the simple current $J^{\prime}$ comes in. Its phase $Q\left(\mu^{\prime}\right)$ is $\frac{\mu_{n}^{\prime}}{2}$ (recall (2.3)), and so the alternating sum in (4.3) projects away $\mu_{n}^{\prime}$ even, as well as $\mu_{0}^{\prime}=\mu_{1}^{\prime}$, and absorbs an extra factor of 2 coming from our formula $\psi=2 S^{\prime}$. The weights with $\mu_{0}^{\prime}<\mu_{1}^{\prime}$ are recovered by taking $J^{\prime}$-orbits of $\mu^{\prime}$, and this absorbs the remaining factor of 2 . We have also used here that $Q\left(\gamma^{\prime}\right) \in \mathbb{Z}$ and $Q\left(a^{\prime}\right), Q\left(b^{\prime}\right) \in \mathbb{Z}+\frac{1}{2}$.

\section{The case of $\mathbf{s u}(2 n)$}

In this case, the $\psi$-matrix is a submatrix of $S$ (rescaled by $\sqrt{2}$ ) for $\widehat{B}_{n}$ level $k+1$, using the identifications $\mu \mapsto\left(\mu_{0}+\mu_{1}+1 ; \mu_{1}, \ldots, \mu_{n}\right) \in P_{+}^{k+1}\left(B_{n}\right)$ and $a \mapsto\left(a_{0} ; a_{1}, \ldots, a_{n-1}, 2 a_{n}+\right.$ 1) $\in P_{+}^{k+1}\left(B_{n}\right)$. But in order to interpret the resulting NIM-rep coefficients $\mathcal{N}_{\lambda a}^{b}$ using ordinary fusions $N^{\prime}$, we need to break the algebra down further.

Write $A_{1}^{n}$ for the direct $\operatorname{sum} \operatorname{su}(2) \oplus \cdots \oplus \operatorname{su}(2)$ ( $n$ times), and put $P_{+}^{\prime}=P_{+}^{k+2 n-2}\left(A_{1}\right)$. Given an exponent $\mu \in \mathcal{E}$, define the weight $\mu^{\prime}=\left(\mu^{\prime(1)}, \ldots, \mu^{\prime(n)}\right) \in P_{+}^{\prime}$ by $\mu^{\prime(i)}=(k+2 n-$ $2-\mu[i] ; \mu[i]) \in P_{+}^{k+2 n-2}\left(A_{1}\right)$. Similarly, define $a^{\prime} \in P_{+}^{\prime}$ by $a^{\prime(i)}=(k+2 n-2-a[i] ; a[i]) \in$ $P_{+}^{k+2 n-2}\left(A_{1}\right)$. The quantities $b_{\gamma^{\prime}}^{\lambda}$ in (4.6) are the $A_{1}^{n} \subset \operatorname{su}(2 n)$ branching coefficients $\oplus_{\gamma^{\prime}} b_{\gamma^{\prime}}^{\lambda}\left(\gamma^{\prime}\right)=(\lambda)$. The first sum in (4.6) is over all $n$-tuples $d \in \mathbb{Z}_{2}^{n}$ with $\sum_{i} d_{i}$ even. $A_{1}$ has a simple current $J^{\prime}$ which sends $\nu^{\prime}$ to $\left(\nu_{1}^{\prime} ; \nu_{0}^{\prime}\right)$, and by $J^{\prime d} \gamma^{\prime}$ we mean the weight with $\left(J^{\prime d} \gamma^{\prime}\right)^{(i)}=J^{\prime d_{i}}\left(\gamma^{\prime(i)}\right)$. The third sum in (4.6) is over all permutations $\pi \in S_{n}$, which act on $a^{\prime}$ by $\left(\pi a^{\prime}\right)^{(i)}=a^{\prime(\pi i)}$. The quantity $\epsilon(\pi)$ is the sign of $\pi$.

Expand out (4.5) into

$$
\psi_{a \mu}=\frac{2^{n-\frac{1}{2}}}{(k+2 n)^{\frac{n}{2}}} \mathrm{i}^{n^{2}-n} \sum_{\pi} \epsilon(\pi) \prod_{i} \sin \left(\frac{\pi a[\pi i] \mu}{k+2 n}\right)=2^{\frac{n-1}{2}} \mathrm{i}^{n^{2}-n} \sum_{\pi} \epsilon(\pi) S_{\pi a^{\prime}, \mu^{\prime}}^{\prime}
$$


The set of all $\mu^{\prime}$ here consist precisely of all the weights in $P_{+}^{\prime}$ with $\mu_{1}^{\prime(1)}>\mu_{1}^{\prime(2)}>\cdots>$ $\mu_{1}^{\prime(n)}$ and $\mu_{1}^{\prime(1)} \equiv \cdots \equiv \mu_{1}^{\prime(n)}(\bmod 2)$. Again, we need to extend the sum over these $\mu^{\prime}$ to a sum over all $P_{+}^{\prime}$. The non-trivial simple current of $A_{1}$ has phase $Q(\nu)=\frac{\nu_{1}}{2}$. Thus the sum in (4.5) over the $2^{n-1} n$-tuples $d$ will retain only those $\mu^{\prime}$ with $\mu_{1}^{\prime(1)} \equiv \cdots \equiv \mu_{1}^{\prime(n)}$ (mod 2 ), and with the numbers $\mu_{1}^{\prime(i)}$ all pairwise distinct. It also absorbs the extra factor of $2^{n-1}$ coming from (5.2). An additional sum over the $S_{n}$-orbits of $\mu^{\prime}$ removes the $\mu_{1}^{\prime(1)}>\mu_{1}^{\prime(2)}>\cdots>\mu_{1}^{\prime(n)}$ restriction. This also cancels an extra factor of $n$ ! coming from our over-counting of fusion coefficients due to the sum over $S_{n}$-orbits of both $a^{\prime}$ and $b^{\prime}-$ we use here the fact that the branching coefficients $b_{\gamma^{\prime}}^{\lambda}$ and $b_{\pi \gamma^{\prime}}^{\lambda}$ are equal, for any $\pi$.

\section{The case of $\operatorname{so}(2 n)$}

Write $P_{+}^{\prime}=P_{+}^{k+1}\left(B_{n-1}\right)$. Under the identification $\mu \mapsto \mu^{\prime}=\left(\mu_{0} ; \mu_{1}, \ldots, \mu_{n-2}, 2 \mu_{n-1}+\right.$ 1) $\in P_{+}^{\prime}$ and $a \mapsto a^{\prime}=\left(a_{0}+a_{1}+1 ; a_{1}, \ldots, a_{n-1}\right) \in P_{+}^{\prime}$, the $\psi$-matrix (4.8) is seen to be a submatrix (up to a factor of $\sqrt{2}$ ) of the $S$-matrix of $\widehat{B}_{n-1}$ at level $k+1$.

Recall that the simple current $J$ of $\widehat{B}_{n-1}$ acts on $\gamma^{\prime}$ by interchanging $\gamma_{0}^{\prime} \leftrightarrow \gamma_{1}^{\prime}$, and has phase $Q\left(\gamma^{\prime}\right)=\frac{\gamma_{n-1}^{\prime}}{2}$. The $\mu^{\prime}$ consist of all weights in $P_{+}^{\prime}$ with $\mu_{n-1}^{\prime}$ odd (i.e. the spinors). The coefficients $b_{\gamma^{\prime}}^{\lambda}$ in (4.9) are the branching rules $\oplus_{\gamma^{\prime} \in P_{+}^{\prime}} b_{\gamma^{\prime}}^{\lambda}\left(\gamma^{\prime}\right)=(\lambda)$ for the embedding $B_{n-1} \subset D_{n}$. Some simple branching rules are $\left(\Lambda_{1}^{\prime}\right)+\left(0^{\prime}\right)=\left(\Lambda_{1}\right),\left(\Lambda_{i}^{\prime}\right)+\left(\Lambda_{i-1}^{\prime}\right)=\left(\Lambda_{i}\right)$ for $1<i<n-1$, and $\left(\Lambda_{n-1}^{\prime}\right)=\left(\Lambda_{n-1}\right)=\left(\Lambda_{n}\right)$; others can be found for instance in [31].

\section{The case of so(8)}

Using the identifications $\mu \mapsto\left(\mu_{0} ; \mu_{2}, 3 \mu_{1}+2\right)$ and $a \mapsto\left(a_{0}+a_{1}+a_{2}+2 ; a_{2}, a_{1}\right)$, the $\psi$-matrix can be seen to be a submatrix of the level $k+2 S$-matrix of $\widehat{G}_{2}$ (rescaled by $\sqrt{3}$ ). It is clear from this interpretation of $\psi$ that the $a^{0}$ row of $\psi$ will be strictly positive. That the $\mu^{0}$ column, corresponding to the $\widehat{G}_{2}$ weight $(k ; 0,2)$, of $\psi$ is strictly positive, is a little more awkward: we need to show

$$
c(2 m+3 n)+c(3 m+n)+c(m-2 n)>c(3 m+2 n)+c(2 m-n)+c(m+3 n),
$$

for all $m=a_{1}+a_{2}+2, n=a_{2}+1$, where here $c(x)=\cos \left(\frac{2 \pi x}{\kappa}\right)$; to do this, reduce it first to the quadratic inequality

$$
c(\delta)^{2}-\left(2 c(\sigma)^{3}-c(\sigma)\right) c(\delta)+\left(4 c(\sigma)^{4}-3 c(\sigma)^{2}\right) \geq 0,
$$

where $\sigma=\frac{m+n}{2}=\frac{a_{1}+2 a_{2}+3}{2}$ and $\delta=\frac{m-n}{2}=\frac{a_{1}+1}{2}$. It can be shown by standard arguments that this final inequality is indeed satisfied for all $a \in \mathcal{B}$. 
To get an interpretation of the NIM-rep coefficients $\mathcal{N}$ in terms of ordinary fusions $N^{\prime}$, we must break $G_{2}$ down to $A_{2}$. In particular, write $P_{+}^{\prime}=P_{+}^{k+3}\left(A_{2}\right)$. Associate the exponent $\mu \in P_{+}^{k}\left(D_{4}\right)$ to the weight $\mu^{\prime}=\left(\mu_{0} ; \mu_{2}, 3 \mu_{1}+\mu_{2}+3\right) \in P_{+}^{\prime}$, and the boundary state $a$ to the weight $a^{\prime}=\left(a_{0}+a_{1}+a_{2}+2 ; a_{2}, a_{1}+a_{2}+1\right) \in P_{+}^{\prime}$. In (4.11), $J^{\prime}$ is the simple current of $A_{2}$, which sends $\nu^{\prime}$ to $\left(\nu_{2}^{\prime} ; \nu_{0}^{\prime}, \nu_{1}^{\prime}\right)$. $C^{\prime}$ there is charge conjugation, which interchanges $\nu_{1}^{\prime} \leftrightarrow \nu_{2}^{\prime}$. The quantities $b_{\gamma^{\prime}}^{\lambda}$ are the $A_{2} \subset D_{4}$ branching coefficients $\oplus_{\gamma^{\prime}} b_{\gamma^{\prime}}^{\lambda}\left(\gamma^{\prime}\right)=(\lambda)$. There are two of these branchings; the one we need comes from $A_{2} \subset G_{2} \subset B_{3} \subset D_{4}$. Many of these branching rules can be found in [31]; some of the more useful ones are $2(0,0) \oplus(1,0) \oplus(0,1)=(1,0,0,0)=(0,0,1,0)=(0,0,0,1)$ and $(1,1) \oplus 3(1,0) \oplus 3(0,1) \oplus$ $2(0,0)=(0,1,0,0)$.

Then (4.10) becomes

$$
\psi_{a \mu}=\sqrt{3} \mathrm{i}\left(S_{a^{\prime} \mu^{\prime}}^{\prime}-S_{a^{\prime} \mu^{\prime}}^{\prime}{ }^{*}\right),
$$

expressing $\psi$ in terms of $A_{2}$ data.

The $\mu^{\prime}$ consist of all weights in $P_{+}^{\prime}$ with $\mu_{1}^{\prime}<\mu_{2}^{\prime}$ and $\mu_{1}^{\prime} \equiv \mu_{2}^{\prime}(\bmod 3)$. The first condition on $\mu^{\prime}$ is removed by the sum of $C^{\prime}$-orbits of $\mu^{\prime}$ - we use the fact that the branching coefficients $b_{\gamma^{\prime}}^{\lambda}$ and $b_{C^{\prime} \gamma^{\prime}}^{\lambda}$ are equal. The second condition on $\mu^{\prime}$ is removed by the sum over $i$ in (4.11) (recall that the phase for $J^{\prime}$ is $Q\left(\nu^{\prime}\right)=\frac{\nu_{1}^{\prime}-\nu_{2}^{\prime}}{3}$ ).

\section{The case of $E_{6}$}

In this case the $\psi$-matrix is a submatrix of the level $k+3 S$-matrix (rescaled by 2 ) of $\widehat{F}_{4}$, using the identifications $\mu \mapsto\left(\mu_{0} ; \mu_{6}, \mu_{3}, 2 \mu_{2}+1,2 \mu_{1}+1\right)$ and $a \mapsto\left(a_{0}+a_{3}+a_{2}+\right.$ $\left.a_{1}+3 ; a_{4}, a_{3}, a_{2}, a_{1}\right)$. Now, any $\widehat{F}_{4}$ level $k$ quantities can be rewritten in terms of $\widehat{\operatorname{so}}(8)$ level $k+3$ ones, using the embedding $\operatorname{so}(8) \subset F_{4}$, and this will enable us to interpret these NIM-reps using $\widehat{\mathrm{sO}}(8)$ fusion coefficients.

In particular, write $P_{+}^{\prime}=P_{+}^{k+6}\left(D_{4}\right)$, and associate the exponent $\mu \in P_{+}^{k}\left(E_{6}\right)$ to the weight $\mu^{\prime}=\left(\mu_{0} ; 2 \mu_{1}+2 \mu_{2}+\mu_{3}+4, \mu_{6}, \mu_{3}, 2 \mu_{2}+\mu_{3}+2\right) \in P_{+}^{\prime}$ and the boundary state $a$ to $a^{\prime}=\left(a_{0}+a_{1}+a_{2}+a_{3}+3 ; a_{1}+a_{2}+a_{3}+2, a_{4}, a_{3}, a_{2}+a_{3}+1\right) \in P_{+}^{\prime}$. The sum in (4.14) over $J^{\prime}$ is over the 4 simple currents of so(8): the identity; $J_{v}^{\prime} \nu^{\prime}=\left(\nu_{1}^{\prime} ; \nu_{0}^{\prime}, \nu_{2}^{\prime}, \nu_{4}^{\prime}, \nu_{3}^{\prime}\right)$; $J_{s}^{\prime} \nu^{\prime}=\left(\nu_{4}^{\prime} ; \nu_{3}^{\prime}, \nu_{2}^{\prime}, \nu_{1}^{\prime}, \nu_{0}^{\prime}\right)$; and $J_{v}^{\prime} \circ J_{s}^{\prime}$. The sum there over $\pi$ is over all 6 conjugations of so(8), corresponding to each of the 6 possible permutations $\pi$ of the 1st, 3rd, and 4th Dynkin labels, and $\epsilon(\pi)$ is the sign of that permutation. The numbers $b_{\gamma^{\prime}}^{\lambda}$ are the $D_{4} \subset E_{6}$ branching coefficients $\oplus_{\gamma^{\prime}} b_{\gamma^{\prime}}^{\lambda}\left(\gamma^{\prime}\right)=(\lambda)$. Some useful $D_{4} \subset E_{6}$ branching rules are $3(0000) \oplus(1000) \oplus(0010) \oplus(0001)=(100000)=(000010)$ and $2(0000) \oplus 2(1000) \oplus$ $(0100) \oplus 2(0010) \oplus 2(0001)=(000001)$. 
To prove (4.14), note first that $\psi_{a \mu}=2 \sum_{\pi} \epsilon(\pi) S_{\pi a^{\prime}, \mu^{\prime}}^{\prime}$, where the sum is over all 6 permutations $\pi$ of the labels $a_{1}^{\prime}, a_{3}^{\prime}, a_{4}^{\prime}$. The weights $\mu^{\prime}$ consist of all weights in $P_{+}^{\prime}$ with $\mu_{1}^{\prime}>\mu_{3}^{\prime}>\mu_{4}^{\prime}$ and $\mu_{1}^{\prime} \equiv \mu_{3}^{\prime} \equiv \mu_{4}^{\prime}(\bmod 2)$. The phases for the simple currents $J_{v}^{\prime}$ and $J_{s}^{\prime}$ are $Q_{v}\left(\nu^{\prime}\right)=\frac{\nu_{3}^{\prime}+\nu_{4}^{\prime}}{2}$ and $Q_{s}\left(\nu^{\prime}\right)=\frac{\nu_{1}^{\prime}+\nu_{3}^{\prime}}{2}$. The rest is as before.

\subsection{Further remarks}

It remains to show that, for the case of the $A$ - and $D$-series, the column of $\psi$ corresponding to $\mu^{0}=(k ; 0, \ldots, 0)$, and the row of $\psi$ corresponding to $a^{0}=(k ; 0, \ldots, 0)$ are both strictly positive. The key observation for the proof of these statements is the fact that $\psi$ is a submatrix of some untwisted $S$-matrix (see section 5.2). In some cases (e.g. for the $\mu^{0}$ column for the case of $\left.\mathrm{su}(2 n)\right)$ strict positivity then follows automatically from the fact that the vacuum row or column of that $S$-matrix is strictly positive (see Remark 13.8 in 25]). However, in most other cases we need to know the sign of the $\Lambda_{n}$ row and column of the $S$-matrix for $\widehat{B}_{n}$.

Consider for concreteness the $\mu^{0}=(k ; 0, \ldots, 0)$ column of $\psi$, for $\operatorname{su}(2 n+1)$. We know from section 4.1 that $\psi_{a \mu^{0}}=2 S_{a^{\prime} \Lambda_{n}^{\prime}}^{\prime}$. The sign of $S_{a^{\prime} \Lambda_{n}^{\prime}}^{\prime}$ equals the sign of $S_{\Lambda_{n}^{\prime} a^{\prime}}^{\prime} / S_{0^{\prime} a^{\prime}}^{\prime}$, which is a value of the $\Lambda_{n}$-character of $B_{n}$. This is easy to calculate, and we find

$$
\frac{S_{\Lambda_{n}^{\prime} a^{\prime}}^{\prime}}{S_{0^{\prime} a^{\prime}}^{\prime}}=2^{n} \prod_{i=1}^{n} \sin \left(\frac{\pi a[i]}{k+2 n+1}\right) .
$$

Given the definition of $a[i]$, we therefore conclude that $\psi_{a^{\prime} \mu^{0}}>0$. All other algebras are handled in the same way.

Finally, let us discuss the simple current symmetries for the various NIM-reps. The simple current $J^{E}$ for su(2n), which corresponds to the Dynkin symmetry of $D_{n}^{(2)}$, permutes $\mu \in \mathcal{E}$ by $J^{E} \mu=\left(\mu_{n} ; \mu_{n-1}, \ldots, \mu_{0}, \mu_{1}, \ldots, \mu_{n-1}\right)$. Its phase $Q^{E}(a)=\frac{1}{2} \sum_{i} i a_{i}$ will be a grading for the NIM-rep coefficients (see (2.16)), where $Q^{E}(\lambda)=\frac{1}{2} \sum_{i=1}^{2 n-1} i \lambda_{i}$. The simple current $J^{B}$ for $\operatorname{su}(2 n)$, which corresponds to the Dynkin symmetry of $A_{2 n-1}^{(2)}$, permutes $a \in \mathcal{B}$ by $a_{0} \leftrightarrow a_{1}$, and has phase $Q^{B}(\mu)=\mu_{n}$. It defines a symmetry of the NIM-rep (see (2.16)).

The simple currents $J^{E}$ and $J^{B}$ for so(2n) are defined similarly, except with their formulae interchanged (for example $J^{E}$ acts on $\mu$ by interchanging $\mu_{0} \leftrightarrow \mu_{1}$, and we have $\left.Q^{E}(a)=a_{n}\right)$. The NIM-rep grading (2.16) arises with the choice $Q^{E}(\lambda)=\frac{1}{2}\left(\lambda_{n-1}+\lambda_{n}\right)$. 


\section{Generalisations}

The above constructions generalise also to certain other classes of modular invariants, in particular those that come from simple currents (2.3). Here we shall only give the details for two simple cases; a complete description will be given elsewhere. Together, these two cases cover all $\operatorname{su}(p)$ level $k$, where $p \geq 3$ is prime. The $\psi$-matrices for all modular invariants of $\mathrm{su}(2)$ were given in [3] ( $\psi$ for the 'tadpole' $\mathrm{su}(2)$ NIM-rep, which does not correspond to a modular invariant, is a special case of the next subsection). The simple current NIM-reps given below are presumably consistent with those in [11], but we are more explicit here. We should also like to emphasise that, unlike us, [11] do not have a rigorous proof that their conjectured NIM-reps are non-negative integers (see the discussion in the introduction).

Consider any $\operatorname{su}(n)$ level $k$. It has a simple current $J$ of order $n$, corresponding to the cyclic symmetry of the extended Dynkin diagram, which sends the highest weight $\lambda=\left(\lambda_{0} ; \lambda_{1}, \ldots, \lambda_{n-1}\right)$ to $J \lambda=\left(\lambda_{n-1} ; \lambda_{0}, \ldots, \lambda_{n-2}\right)$. For any divisor $d$ of $n$, define the matrix $M[d]$ by

$$
M[d]_{\lambda, \mu}=\sum_{j=1}^{n / d} \delta^{\frac{n}{d}}\left(t(\lambda)+\frac{d j k^{\prime}}{2}\right) \delta_{\mu, J^{j d} \lambda},
$$

where $t(\lambda)=\sum_{i} i \lambda_{i}$, and $k^{\prime}=k+n$ or $k^{\prime}=k$ depending on whether or not both $k$ and $n$ are odd, respectively. In (6.1) we write $\delta^{y}(x)=0$ or 1 depending, respectively, on whether or not $\frac{x}{y} \in \mathbb{Z}$. Then $M[d]$ is a modular invariant if and only if the product $(n-1) k d$ is even. For instance, $M[n]=I$ is a modular invariant for any $\operatorname{su}(n)$ level $k$; for $\operatorname{su}(2), M[1]$ is a modular invariant if and only if $k$ is even.

\subsection{A case without fixed points}

Let us consider $\operatorname{su}(n)$ level $k$, for the simple current $J$, when $\operatorname{gcd}(k, n)=1$. We will explicitly give below the corresponding $\psi$-matrix and prove it yields a NIM-rep (as always, the hardest thing to prove is that the entries $\mathcal{N}_{\lambda x}^{y}$ are non-negative integers). For such $\operatorname{su}(n)$ level $k$, the matrix $M[1]$ in (6.1) will be a modular invariant if and only if $n$ is odd. However, the matrices defined by the $\psi$-matrix given below define a NIM-rep even if this is not the case. For example, if $n=2$ and $k$ is odd, they describe the 'tadpole graph' of [3]. So for $n$ even, this NIM-rep generalises the tadpole.

Let $P_{0}$ be all level $k$ weights $\mu=\left(\mu_{1}, \ldots, \mu_{n-1}\right)$ of su $(n)$, with $n$ dividing $t(\mu)=\sum_{i} i \mu_{i}$. Then $P_{0}$ will label both the rows and columns of $\psi$. (We see explicitly in (6.1) that 
$M[1]_{\mu \mu} \neq 0$ if and only if $\mu \in P_{0}$.) The matrix $\psi$ is actually the submatrix of the usual $S$-matrix, restricted to $P_{0}$, and then rescaled by $\sqrt{n}$. More specifically, it is given by

$$
\psi_{\mu \nu}=c \exp \left[2 \pi \mathrm{i} \frac{t^{+}(\mu) t^{+}(\nu)}{(n+k) n}\right] \operatorname{det}\left[\exp \left(-2 \pi \mathrm{i} \frac{\mu^{+}[i] \nu^{+}[j]}{n+k}\right)\right]_{1 \leq i, j \leq n}
$$

where

$$
c=(n+k)^{\frac{-n+1}{2}} \mathrm{i} \frac{n(n-1)}{2},
$$

and as before $t^{+}(\mu)=\frac{n(n-1)}{2}+\sum_{i} i \mu_{i}$, and $\mu^{+}[i]=n-i+\sum_{j=i}^{n-1} \mu_{j}$.

For any $\lambda \in P_{+}$and $\mu, \nu \in P_{0}$, this NIM-rep coefficient $\mathcal{N}_{\lambda \mu}^{\nu}$ equals the fusion coefficient $N_{J^{i} \lambda \mu}^{\nu}$, where $i$ is the unique solution $(\bmod n)$ to the congruence $k i+t(\lambda) \equiv 0$. To see this, note that this $i$ is the unique number for which $J^{i} \lambda \in P_{0}$. The $S$-matrix factorises into the tensor product of the matrix $\psi$ in (6.2), and a $\mathrm{U}(1)$ level $n S$-matrix of phases. Now, whenever the $S$-matrix decomposes into a tensor product, then the fusions decompose into a product. So the $\operatorname{su}(n)$ level $k$ fusion $\lambda \times \mu$ here will be $J^{-i}\left(\left(J^{i} \lambda\right) \times \mu\right)$, where the fusion $\left(J^{i} \lambda\right) \times \mu$ of weights in $P_{0}$ can be obtained by putting $\psi$ directly into Verlinde's formula (2.2). This means that (2.12) here will equal $N_{J^{i} \lambda \mu}^{\nu}$, as desired.

Hence we have proven that this choice of $\psi$ will always define a (non-negative integer) NIM-rep with the correct exponents. A similar analysis applies for instance to so $(2 n+1)$, so $(2 n)$ and $\operatorname{sp}(4 n+2)$, when the level $k$ is odd.

\subsection{A case with fixed points}

Next, let us consider $\operatorname{su}(p)$ with $p \geq 2$ prime, when $p$ divides the level $k$ (for $p=2$ we require 4 to divide $k$ ). As we shall see shortly, that the formula we are about to give is indeed a NIM-rep follows from the conjectured formula in Section 6 of [32] for the $S$-matrix $S^{e}$ of the chiral extension by a simple current, as is also for example mentioned in [11]. As long as $p^{2}$ does not divide $k+p$, we can actually prove that our $\psi$ matrix yields a (non-negative integer) NIM-rep.

The exponents are the weights $\mu=\left(\mu_{0} ; \mu_{1}, \ldots, \mu_{n-1}\right)$ with $p$ dividing $t(\mu)=\sum_{i} i \mu_{i}$. There is a single fixed point $\phi=\left(\frac{k}{p} ; \frac{k}{p}, \ldots, \frac{k}{p}\right)$, which has multiplicity $p$ (as an exponent). The 'boundary states' consist of all $J$-orbits $[\nu]$. Again, the fixed point $\phi$ has multiplicity p. Although it may not be completely obvious, these two sets always have the same cardinality, namely

$$
\frac{1}{p}\left(\frac{(n+p-1) !}{(n-1) ! p !}+p^{2}-1\right)
$$


Define $\psi_{\mu,[\nu]}$, when neither $\mu$ nor $\nu$ are fixed points by

$$
\psi_{\mu,[\nu]}=\sqrt{p} S_{\mu \nu}
$$

where $S$ is the $S$-matrix of $\operatorname{su}(p)$ level $k$. Of course this formula is independent of which representative $\nu$ is taken for the $J$-orbit $[\nu]$.

When $\mu$ is the fixed point $\phi$ but $\nu$ is not, then

$$
\psi_{(\phi, i),[\nu]}=S_{\phi \nu}
$$

for any $i=0, \ldots, p-1$.

When $\mu$ is not the fixed point, but $\nu=\phi$, then

$$
\psi_{\mu,([\phi], j)}=\frac{S_{\mu \phi}}{\sqrt{p}}
$$

for any $j$.

Finally, when both $\mu=\nu=\phi$, we get for $p$ odd

$$
\psi_{(\phi, h),([\phi], j)}=\frac{1}{p}\left(x-1+p \delta_{h, j}\right)
$$

where

$$
x=\left(\frac{p}{k+p}\right)^{\frac{p-1}{2}} s \quad \text { for the Legendre symbol } \quad s=\left(\frac{(k+p) / p}{p}\right) .
$$

Recall that the Legendre symbol $\left(\frac{(k+p) / p}{p}\right)$ equals 0 if $p^{2}$ divides $k+p$; otherwise it equals \pm 1 if $(k+p) / p$ is or is not a perfect square $\bmod p$, respectively. So $\left(\frac{a}{3}\right)=1,0,-1$ for $a \equiv 1,0,2(\bmod 3)$, respectively, whereas $\left(\frac{a}{5}\right)=1,0,-1$ for $a \equiv \pm 1, a \equiv 0, a \equiv \pm 2(\bmod$ $5)$, respectively.

On the other hand, for $p=2$, the formula becomes

$$
\psi_{(\phi, h),([\phi], j)}=\frac{1}{2}\left[(-1)^{\frac{k}{4}} \sqrt{\frac{2}{k+2}}+\mathrm{i}^{\frac{k}{4}}(-1)^{h+j}\right] .
$$

In this special case, the formula agrees with Eq. (B.6) of [3].

In order to calculate with and analyse this $\psi$ matrix, it is convenient to use 'fixed-point factorisation' 33. This is a way of computing $S$-matrix entries involving simple current 
fixed points. For instance the entries $S_{\phi \nu}$ will equal either 0 or $\pm\left(\frac{p}{k+p}\right)^{\frac{p-1}{2}}$. The quantity $x$ in (6.9) equals $S_{\phi \phi}$. From this we quickly get that our $\psi$ will indeed be unitary.

It is now straightforward to calculate the NIM-rep coefficients $\mathcal{N}_{\lambda x}^{y}$ in terms of the WZW fusion rules. We find (whenever $\mu, \nu$ are not fixed points)

$$
\begin{aligned}
\mathcal{N}_{\lambda[\mu]}^{[\nu]} & =\sum_{J} N_{J \lambda \mu}^{\nu} \\
\mathcal{N}_{\lambda[\phi, i]}^{[\nu]} & =N_{\lambda \phi}^{\nu} \\
\mathcal{N}_{\lambda[\phi, i]}^{[\phi, j]} & =\frac{1}{p}\left(N_{\lambda \phi}^{\phi}+\left(p \delta_{i j}-1\right) \frac{S_{\lambda \phi}}{S_{0 \phi}}\right) .
\end{aligned}
$$

Thus the first two are manifestly non-negative integers. We know (from fixed-point factorisation) that the ratio $\frac{S_{\lambda \phi}}{S_{0 \phi}}$ here will be 0 or \pm 1 . So it suffices to verify, for all $\lambda \in P_{+}$, that

$$
N_{\lambda \phi}^{\phi} \equiv \frac{S_{\lambda \phi}}{S_{0 \phi}}(\bmod p)
$$

Condition (6.12) is certainly true when $p$ does not divide $t(\lambda)$ - in that case we have $N_{\lambda \phi}^{\phi}=0=\frac{S_{\lambda \phi}}{S_{0 \phi}}$. When $p$ does divide $t(\lambda)$, then our $\mathcal{N}_{\lambda[\phi, i]}^{[\phi, j]}$ precisely equals the extended fusion coefficient $N_{[\lambda][\phi, i]}^{e[\phi, j]}$, for the conjectured $S$-matrix $S^{e}$ for the simple current chiral extension, as given in [32]. This observation is also made in [11]. So provided their conjecture [32] is correct (as is expected), then the $\psi$ given above does indeed define a (nonnegative integer) NIM-rep. Some relation between NIM-reps and extended $S$-matrices is expected - see e.g. section 6 of [23], Thm. 4.16 of [16], and (B.6) in [3].

Assume now that $p^{2}$ does not divide $p+k$. Then $p$ does not divide the integer $\frac{1}{S_{0 \phi}^{2}}=\left(\frac{k}{p}+1\right)^{p-1}$. So it suffices to show that $p$ must divide the integer

$$
\frac{1}{S_{0 \phi}^{2}}\left(N_{\lambda \phi}^{\phi}-\frac{S_{\lambda \phi}}{S_{0 \phi}}\right)=\sum_{\mu} \frac{S_{\lambda \mu} S_{\phi \mu}^{2}}{S_{0 \mu} S_{0 \phi}^{2}}+\frac{S_{\lambda \phi}}{S_{0 \phi}}\left(\frac{S_{\phi \phi}^{2}}{S_{0 \phi}^{2}}-\frac{1}{S_{0 \phi}^{2}}\right)
$$

where the sum is over all non-fixed-points $\mu$.

Consider the sum

$$
\sum_{\mu} \frac{S_{\lambda \mu} S_{\phi \mu}^{2}}{S_{0 \mu} S_{0 \phi}^{2}}=p \sum_{[\mu]} \frac{S_{\lambda \mu}}{S_{0 \mu}}
$$

where the first sum is over all non-fixed-points $\mu$, and the second sum is over all $J$-orbits of non-fixed-points $\mu$ with $S_{\mu \phi} \neq 0$. Note that this sum over $[\mu]$ will be an algebraic integer (since each ratio $\frac{S_{\lambda \mu}}{S_{0 \mu}}$ is an algebraic integer, being the eigenvalue of an integer matrix) 
and it will be rational (since it will be fixed by all Galois automorphisms), and therefore it must be an integer. So $p$ divides the first term on the right hand side of (6.13).

Also, Fermat's Little Theorem implies that $p$ must divide the integer

$$
\frac{S_{\phi \phi}^{2}}{S_{0 \phi}^{2}}-\frac{1}{S_{0 \phi}^{2}}=1-\left(\frac{k}{p}+1\right)^{p-1}
$$

Thus $p$ divides (6.13), and hence $\mathcal{N}$ will indeed be a non-negative integer, provided only that $p$ does not divide $\frac{k}{p}+1$.

\section{Conclusion}

In this paper we have made a proposal for the boundary states of the WZW models that correspond to the conjugation modular invariant associated to a symmetry $\omega$ of the unextended Dynkin diagram. The boundary states of this theory are labelled by the $\omega$ twisted representations, and they are described by a formula (3.13) similar to Cardy's expression for the boundary states of the diagonal theory. The corresponding NIM-rep agrees precisely with the twisted fusion rules. These fusion rules are given by a Verlindelike formula (3.14).

We have given explicit formulae for the $\psi$-matrix for all possible cases (section 4 ), and we have related the NIM-rep entries to untwisted fusion rules, thereby proving their integrality. We have also checked nonnegativity explicitly for numerous cases. Some of the corresponding fusion graphs have been collected in the appendix.

Our formulae seem to generalise further to certain other classes of modular invariants, in particular those associated to simple currents. In order to illustrate this point, we have described two classes in detail in section 6 . The results presented there already suffice to determine the NIM-reps for all simple current modular invariants for $\mathrm{su}(p)$ level $k$, when $p \geq 3$ is prime. We have also proven that our NIM-rep coefficients are non-negative integers (at least when $p^{2}$ does not divide $k+p$ ). The general case (that will be described elsewhere) will essentially cover all remaining modular invariant WZW models.

\section{Acknowledgements}

We are grateful to David Evans, Peter Goddard, Valya Petkova, Andreas Recknagel and Jean-Bernard Zuber for useful conversations and communications. We thank Jürgen Fuchs, 
Christoph Schweigert, and Bert Schellekens for explaining their work to us. We also thank Thomas Mettler for drawing our attention to a number of small mistakes in the published version which are corrected here. MRG is grateful to the Royal Society for a University Research Fellowship. He also acknowledges partial support from the EU network 'Superstrings' (HPRN-CT-2000-00122), as well as from the PPARC special grant 'String Theory and Realistic Field Theory', PPA/G/S/1998/0061. TG warmly thanks St. John's College, Cambridge, which generously supported him in the early stages of this project; his research is supported in part by NSERC.

\section{Appendix A. Explicit descriptions of the fusion graphs}

In this appendix we give explicit descriptions of some of the NIM-reps we have found in terms of the corresponding graphs. For the examples under consideration, the NIMreps are often characterised in terms of the matrix associated to the field $\lambda=\Lambda_{1}$. The corresponding graph has $M$ vertices labelled by the rows (or columns) of $\mathcal{N}_{\lambda}$, and the vertex associated to $i$ and $j$ are linked by $\left(\mathcal{N}_{\lambda}\right)_{i j}$ lines. Incidentally, given our discussion of section 3 above, these graphs are precisely the fusion graphs that describe the fusion of the field $\lambda$ with the twisted representations (that label the rows and columns of $\mathcal{N}_{\lambda}$ ). In particular, the NIM-rep matrices considered here are therefore symmetric, and the relevant graphs are not oriented.

For the case of $\mathrm{su}(n)$ with $\omega$ being charge conjugation, the graphs are explicitly given in Figure 1. The left-right symmetry of the su(4) graphs is due to the symmetry $J^{B}$ of the $A_{3}^{(2)}=D_{3}^{(2)}$ Dynkin diagram. In addition, the graphs for $\mathrm{su}(4)$ are 2-colourable because of the symmetry $J^{E}$ of the $D_{3}^{(2)}$ diagram. Finally, there is also a left-right symmetry of the $\mathrm{su}(3)$ and $\mathrm{su}(5)$ graphs when the level is odd, due to the $C_{1}^{(1)}=A_{1}^{(1)}$ and $C_{2}^{(1)}$ Dynkin symmetries $J^{B}$, respectively.

For the case of so $(2 N)$ with chirality flip, the relevant graphs are explicitly given in Figure 2. The graphs for so(6) do not agree with those of $\mathrm{su}(4)$ given in Figure 1 since, under the identification $\mathrm{su}(4) \cong \mathrm{so}(6), \Lambda_{1}$ of $\mathrm{su}(4)$ corresponds to $\Lambda_{3}$ of $\mathrm{so}(6)$. All of these graphs have an order-2 symmetry, which is due to the symmetry $J^{B}$ of the $D_{3}^{(2)}$ and $D_{4}^{(2)}$ Dynkin diagrams. The graphs are disconnected because of the symmetry $J^{E}$ of the $A_{3}^{(2)}$ and $A_{4}^{(2)}$ diagrams. In particular $Q^{E}\left(\Lambda_{1}\right)=0$, and thus the grading (2.16) implies that each component consists of the $a \in \mathcal{B}$ with a fixed value of the grade $Q^{E}(a)$. 
0
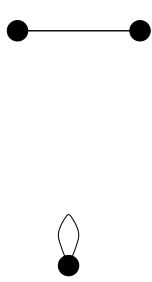
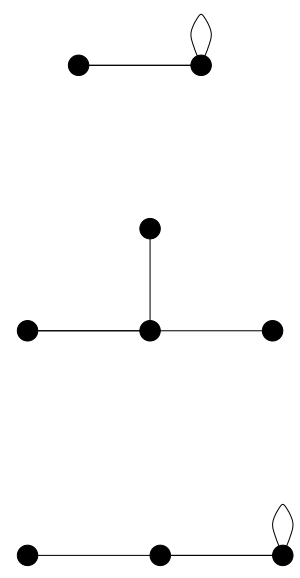
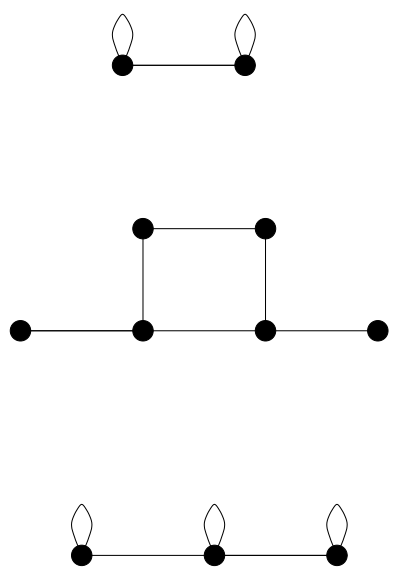

Fig. 1: The NIM-rep graphs for $\mathrm{su}(N)$ with charge conjugation for $N=3,4,5$ and $k=1,2,3$.
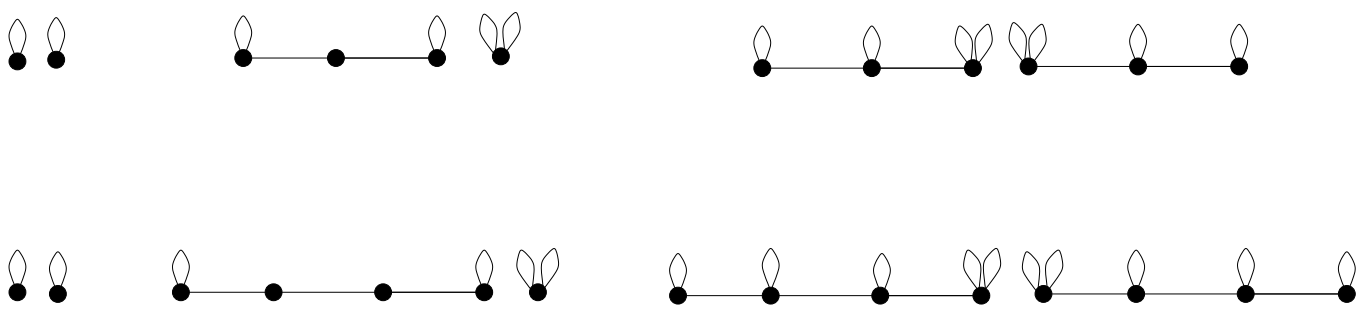

Fig. 2: The NIM-rep graphs for so(2N) with chirality flip for $N=3,4, k=1,2,3$.

For the case of so(8) with triality, and the case of $E_{6}$ with charge conjugation, the relevant graphs are explicitly given in Figure 3 and Figure 4. respectively.
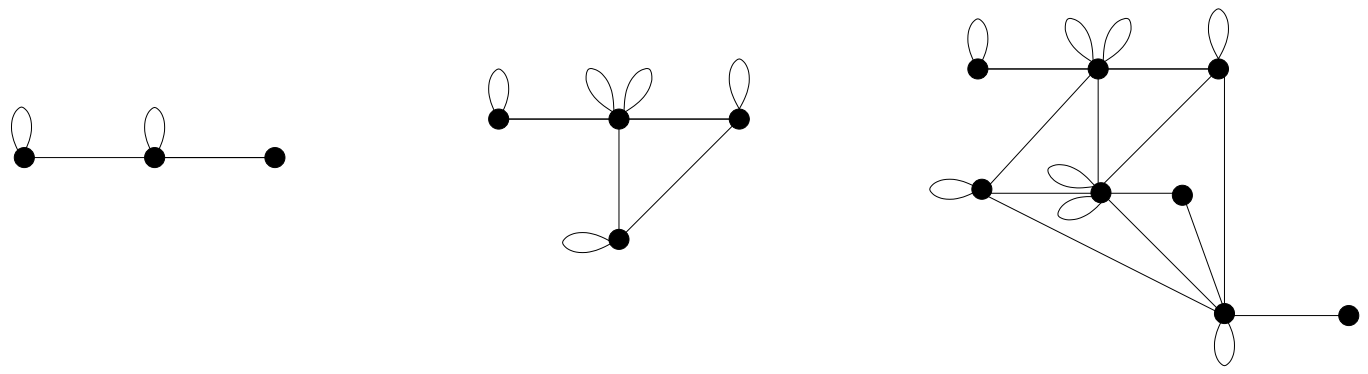

Fig. 4: The NIM-rep graphs for $E_{6}$ with charge conjugation for $k=2,3,4$. 

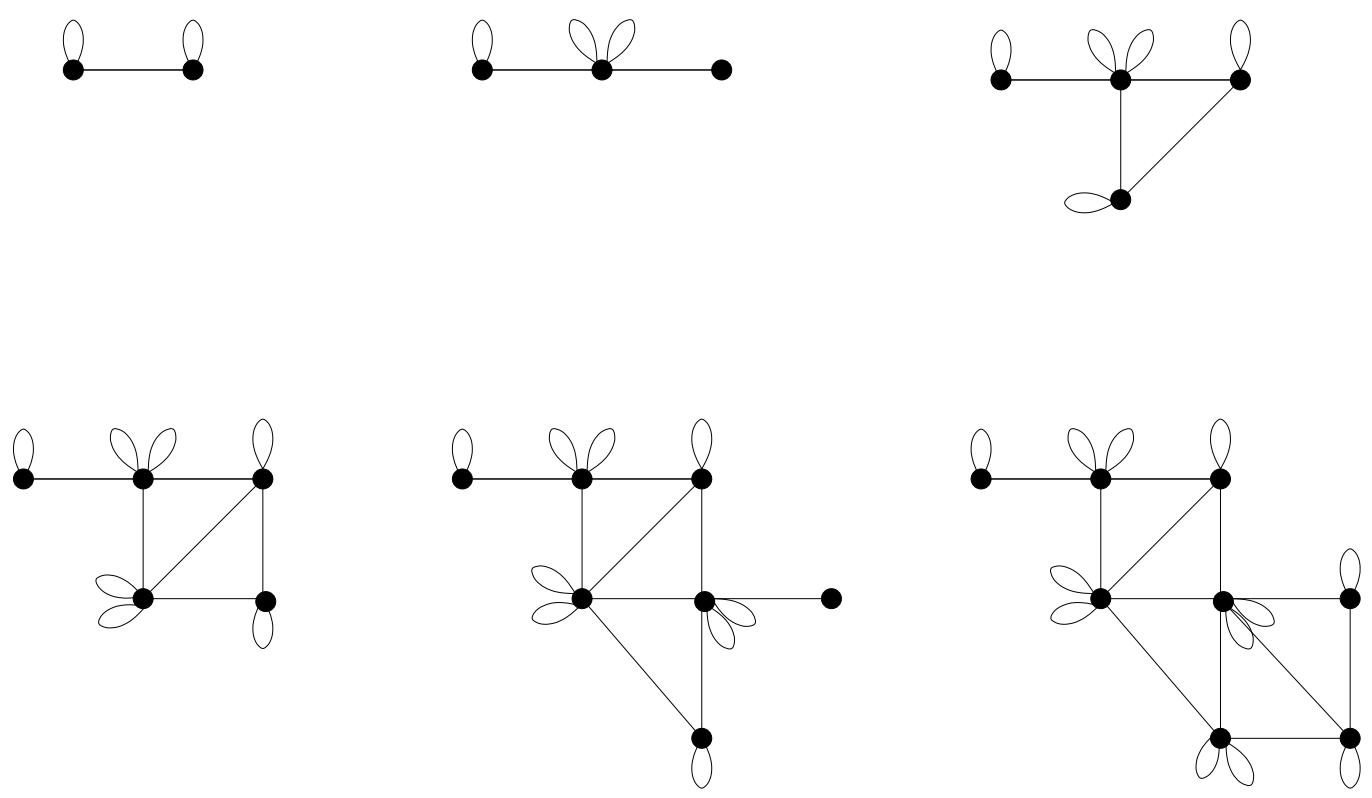

Fig. 3: The NIM-rep graphs for so(8) with triality for $k=2,3,4,5,6,7$. 


\section{References}

[1] J.L. Cardy, Boundary conditions, fusion rules and the Verlinde formula, Nucl. Phys. B324 (1989) 581.

[2] G. Pradisi, A. Sagnotti, Y.S. Stanev, Completeness conditions for boundary operators in 2d conformal field theory, Phys. Lett. B381 (1996) 97; hep-th/9603097.

[3] R.E. Behrend, P.A. Pearce, V.B. Petkova, J.-B. Zuber, Boundary conditions in rational conformal field theories, Nucl. Phys. B579 (2000) 707; hep-th/9908036.

[4] T. Gannon, Modular data: the algebraic combinatorics of conformal field theory, math.QA/0103044.

[5] T. Gannon, Boundary conformal field theory and fusion ring representations, hepth/0106105.

[6] V.B. Petkova, J.-B. Zuber, Conformal field theories, graphs and quantum algebras; hep-th/0108236.

[7] J. Böckenhauer, D.E. Evans, Modular invariants, graphs and $\alpha$-induction for nets of subfactors. III, Commun. Math. Phys. 205 (1999) 183; hep-th/9812110.

[8] A. Ocneanu, talk, Kyoto (2000).

[9] L. Birke, J. Fuchs, C. Schweigert, Symmetry breaking boundary conditions and WZW orbifolds, Adv. Theor. Math. Phys. 3 (1999) 671; hep-th/9905038.

[10] J. Fuchs, C. Schweigert, Solitonic sectors, alpha-induction and symmetry breaking boundaries, Phys. Lett. B490 (2000) 163; hep-th/0006181.

[11] J. Fuchs, L. Huiszoon, B. Schellekens, C. Schweigert, J. Walcher, Boundaries, crosscaps and simple currents, Phys. Lett. B495 (2000) 427; hep-th/0007174.

[12] M. Müger, Conformal Field Theory and Doplicher-Roberts Reconstruction, Fields Inst. Commun. 30 (2001) 297; math-ph/0008027.

[13] P. Di Francesco, J.-B. Zuber, SU(N) lattice integrable models associated with graphs, Nucl. Phys. B338 (1990) 602.

[14] V. Petkova, J.-B. Zuber, From CFT to graphs, Nucl. Phys. B463 (1996) 161; hepth/9510175.

[15] F. Xu, New braided endomorphisms from conformal inclusions, Commun. Math. Phys. 192 (1998) 349.

[16] J. Böckenhauer, D.E. Evans, Y. Kawahigashi, Chiral structure of modular invariants for subfactors, Commun. Math. Phys. 210 (2000) 733; nath.oa/9907149.

[17] V.B. Petkova, J.-B. Zuber, Boundary conditions in charge conjugate sl(N) WZW theories, hep-th/0201239.

[18] H. Ishikawa, Boundary states in coset conformal field theories, hep-th/0111230.

[19] Y. Zhu, Vertex operator algebras, elliptic functions and modular forms, J. Amer. Math. Soc. 9 (1996) 237. 
[20] E. Verlinde, Fusion rules and modular transformations in 2D conformal field theory, Nucl. Phys. B300 (1988) 360.

[21] A.N. Schellekens, S. Yankielowicz, Extended chiral algebras and modular invariant partition functions, Nucl. Phys. B327 (1989) 673.

[22] K. Intriligator, Bonus Symmetry in Conformal Field Theory, Nucl. Phys. B332 (1990) 541.

[23] J. Fuchs, C. Schweigert, Symmetry breaking boundaries I. General theory, Nucl. Phys. B558 (1999) 419; hep-th/9902132.

[24] J. Fuchs, B. Schellekens, C. Schweigert, From Dynkin diagram symmetries to fixed point structures, Commun. Math. Phys. 180 (1996) 39; hep-th/9506135.

[25] V.G. Kac, Infinite dimensional Lie algebras, Cambridge University Press (1990) [3rd ed.].

[26] P. Goddard, D.I. Olive, Kac-Moody and Virasoro algebras in relation to quantum physics, Int. Journ. Mod. Phys. A1 (1986) 303.

[27] M.R. Gaberdiel, Fusion of twisted representations, Int. Journ. Mod. Phys. A12 (1997) 5183 ; hep-th/9607036.

[28] I. Affleck, A.W. Ludwig, Universal noninteger 'ground state degeneracy' in critical quantum systems, Phys. Rev. Lett. 67 (1991) 161.

[29] T. Gannon, Algorithms for affine Kac-Moody algebras, hep-th/0106123.

[30] R. Slansky, Group theory for unified model building, Phys. Rep. 79 (1981) 1.

[31] W.G. McKay, J. Patera, Tables of dimensions, indices, and branching rules for representations of simple Lie algebras, Marcel Dekker Inc., New York (1981).

[32] J. Fuchs, B. Schellekens, C. Schweigert, A matrix $S$ for all simple current extensions, Nucl. Phys. B473 (1996) 323; hep-th/9601078.

[33] T. Gannon, M.A. Walton, On fusion algebras and modular matrices, Commun. Math. Phys. 206 (1999) 1; q-alg/9709039. 